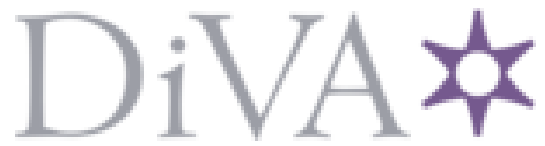

http://www.diva-portal.org

Preprint

This is the submitted version of a paper published in Annual Reviews in Control.

Citation for the original published paper (version of record):

Heshmati-Alamdari, S., Bechlioulis, C P., Karras, G C., Nikou, A., Dimarogonas, D V. et al. (2018)

A robust interaction control approach for underwater vehicle manipulator systems Annual Reviews in Control, 46: 315-325

https://doi.org/10.1016/j.arcontrol.2018.10.003

Access to the published version may require subscription.

N.B. When citing this work, cite the original published paper.

Permanent link to this version:

http://urn.kb.se/resolve?urn=urn:nbn:se:kth:diva-240790 


\title{
A Robust Interaction Control Approach for Underwater Vehicle Manipulator Systems
}

\author{
Shahab Heshmati-Alamdari ${ }^{\mathrm{a}, *}$, Charalampos P. Bechliouliss ${ }^{\mathrm{a}}$, George C. Karras ${ }^{\mathrm{a}}$, Alexandros Nikou ${ }^{\mathrm{b}}$, Dimos V. Dimarogonas ${ }^{\mathrm{b}}$ \\ Kostas J. Kyriakopoulos ${ }^{\mathrm{a}}$ \\ ${ }^{a}$ Control Systems Lab, Department of Mechanical Engineering, National Technical University of Athens, 9 Heroon Polytechniou Street, Zografou 15780.
E-mail: $\{$ shahab, chmpechl, karrasg, kkyria\} @mail.ntua.gr
${ }^{b}$ KTH Center for Autonomous Systems, KTH Royal Institute of Technology, SE-100 44, Stockholm, Sweden. E-mail: $\{$ anikou, dimos\}@kth.se
}

\begin{abstract}
In underwater robotic interaction tasks (e.g., sampling of sea organisms, underwater welding, panel handling, etc) various issues regarding the uncertainties and complexity of the robot dynamic model, the external disturbances (e.g., sea currents), the steady state performance as well as the overshooting/undershooting of the interaction force error, should be addressed during the control design. Motivated by the aforementioned considerations, this paper presents a force/position tracking control protocol for an Underwater Vehicle Manipulator System (UVMS) in compliant contact with a planar surface, without incorporating any knowledge of the UVMS dynamic model, the exogenous disturbances or the contact stiffness model. Moreover, the proposed control framework guarantees: (i) certain predefined minimum speed of response, maximum steady state error as well as overshoot/undershoot concerning the force/position tracking errors, (ii) contact maintenance and (iii) bounded closed loop signals. Additionally, the achieved transient and steady state performance is solely determined by certain designer-specified performance functions/parameters and is fully decoupled from the control gain selection and the initial conditions. Finally, both simulation and experimental studies clarify the proposed method and verify its efficiency.
\end{abstract}

Keywords: Underwater Vehicle Manipulator Systems, Nonlinear Control, Autonomous Underwater Vehicles, Marine Robotics, Force/position Control, Robust Control.

\section{Introduction}

During the last decades, unmanned underwater vehicles are being used widely in various areas such as marine science (e.g., biology, oceanography, archeology) and offshore industry (e.g., 25 5 ship maintenance, inspection of oil/gas facilities, cable burial, mating of underwater connectors, underwater welding) [1]. In particular, a vast number of the aforementioned applications demand the underwater vehicle to be enhanced with intervention capabilities as well [2, 3], thus raising increasing interest on 30 10 Underwater Vehicle Manipulator Systems (UVMSs) [4, 5]. A UVMS consists of an underwater robotic vehicle equipped with robotic manipulators. Currently, underwater intervention is carried out by Remotely Operated Vehicles (ROVs) equipped with one or multiple manipulators, which allow it to grasp, trans- 35 15 port and manipulate objects. However, most ROVs are controlled by a human pilot on a surface ship, via a master-slave tele-operation scheme [6, 7, 8]. Therefore, the well-known disadvantages of human-robot tele-operation (e.g., time delays and increase of human fatigue over time) necessitate for automated and efficient solutions to reduce costs and safety risks, which ${ }^{40}$

\footnotetext{
${ }^{*}$ Corresponding author

Email addresses: shahab@mail .ntua.gr (Shahab Heshmati-Alamdari), chmpechl@mail.ntua.gr (Charalampos P. Bechlioulis),

karrasg@mail.ntua.gr (George C. Karras), anikou@kth.se (Alexandros Nikou), dimos@kth.se (Dimos V. Dimarogonas), kkyria@mail.ntua.gr (Kostas J. Kyriakopoulos)
}

led inevitably to the newal of ROVs with Autonomous Underwater Vehicles (AUVs) as well as to the development of autonomous intervention control platforms like the UVMSs that have attracted significant scientific interest during the last years [9, 4, 10]. Specifically, during the late 90s, efforts on the design of UVMSs were made within the pioneering AMADEUS project [11], which were later exploited in UNION [12] and SAUVIM projects [9], where autonomous underwater intervention was carried out for the first time. A more recent European project which has boosted the underwater robotic interaction with relevant results was TRIDENT [13, 14, 15, 16, 17, 18], where a vehicle-arm system was controlled in a coordinated manner. Another important milestone was achieved in the PANDORA project [19, 20, 21], where a strong emphasis was given on the issue of persistent autonomy. The latest related project in the domain of underwater intervention is the on-going European project DexROV [22], which focuses on inspection and maintenance tasks in the presence of communication latencies.

It is well known that underwater tasks are very challenging owing mainly to external disturbances (i.e., sea currents), the lack of appropriate and adequately accurate sensing/localization [23] and the unknown (or partially known) constrained environment (e.g., offshore industry, oil/gas facilities) [24, 25]. The aforementioned difficulties make the control of underwater manipulator systems a challenging problem that has already gained significant scientific attention within the marine robotic community during the last years [25]. Such con- 
trol problems involve constrained high-dimensional nonlinear ${ }_{105}$ systems with significant complexity regarding the uncertainty ous operational constraints (e.g., visibility constraints and joint limits), the nonlinear coupled dynamics between the underwater vehicle and manipulator systems [26] as well as the grav-110 ity/buoyancy forces that affect the response of the manipulator.

55 All aforementioned challenges should be taken into consideration when designing the control system of a UVMS. Hence, from a control perspective, achieving such goals highlights the need of employing appropriate methodologies from nonlinear ${ }_{115}$ robot control theory [27]. However, most control schemes that

60 have been developed so far for static manipulators and space robots cannot be used directly on UVMS owing to the aforementioned specifics. Therefore, the control of UVMS remains still a challenging task.

Until the early 1990s, only a few research studies had ap-

65 peared that dealt with the UVMS control [28]. Even deriving the dynamic model and developing an efficient dynamic simulation for underwater vehicles equipped with robotic manipulators were studied recently in [29, 30, 31]. However, during the ${ }_{125}$ last years, owing to the enormous demand for more dexterous

70 and efficient underwater interaction robotic platforms, many research studies have been conducted towards enhancing the efficacy of UVMSs [6]. In [32], a hybrid position/force control scheme was designed and implemented on a hydraulically ac- ${ }_{-130}$ tuated manipulator. That work was extended later into a hybrid

75 position/force controller and was compared to a fixed-gain version for various contact stiffness models [33]. However, the dynamic coupling between the manipulator and the vehicle was not considered. In [34], a control scheme was proposed in order ${ }_{135}$ to achieve high maneuverability of an underwater manipulator so mounted on a ROV. A force/torque sensor was assumed to be installed between the ROV and the manipulator to compensate the dynamic effects of the underwater manipulator motion on the vehicle. The control strategy proposed in [35] compensates ${ }_{140}$ for the force/moment exerted on the vehicle from the manip${ }_{85}$ ulator by utilizing the buoyant and gravitational forces, while simultaneously maintaining the contact between the robot endeffector and the environment. In the same direction, the force control strategy proposed in [36] aims at stabilizing the vehi-145 cle when the manipulator operates in a constrained workspace.

90 Moreover, an impedance control technique was studied in [37], where the UVMS dynamic model was considered as an integrated system. That work was extended later in [38], where impedance control was combined with hybrid position/force ${ }_{150}$ control by means of fuzzy switching. Nevertheless, all aforementioned control strategies request accurate knowledge of the contact stiffness model.

On the other hand, treating a UVMS as an integrated system raises significant issues regarding the redundancy of the system, 155 the uncertainties and the dynamic couplings, which all should be considered during the control design. It is well known that a UVMS is kinematically redundant owing to the vehicle's degrees of freedom [39]. The redundancy of a UVMS might be exploited in order to coordinate the system in such a way that ${ }_{160}$ the end-effector tracking accuracy is guaranteed, and this may be achieved by addressing various issues such as: i) the sluggish response of vehicle's thrusters [40, 41, 42], ii) the manipulator's joint limits, iii) the manipulability and iv) the configuration singularities [39]. In general, two strategies at the kinematic [43] and the dynamic [44] levels respectively have been developed for handling the robot's redundancy. The former was enhanced later in [45] for an arbitrary number of priority levels with inferior computational complexities. That framework, which is based on the least-squares minimization technique, solves a finite number of prioritized secondary tasks (e.g., maintaining manipulator's joint limits, increasing the manipulability) in a hierarchical structure. However, a compromise raises among the tasks corresponding to the inequality objectives (e.g., joint limits) [46]. In order to tackle this issue, a method was proposed based on quadratic programming in [47], which solves equality and inequality constraints at any priority level. However, that approach leads in high dimensional quadratic programming problems and cannot handle the activation or deactivation of tasks [48]. The aforementioned strategy was extended later in [49], where instead of a cascade of quadratic programming problems, a single problem is solved that identifies the active set of all the constraints at the same time. Nevertheless, that strategy lacks the ability of enabling and disabling inequality tasks without causing discontinuities. Recently, a generic method that allows activating and deactivating tasks without incurring discontinuities was proposed in [48]. For the case of a UVMS, based on results initially proposed in [50], the redundancy resolution has been employed firstly in [39] to avoid the manipulator's singular configurations. Later, a classical gradient projection method [43] was adopted that addressed various practical criteria such as minimization of load [51, 52] and restoring moments [53]. Finally, a method based on a fuzzy switching technique was proposed in [54] to overcome the conflict among various secondary tasks.

When a UVMS interacts with the environment via its endeffector, the redundancy resolution formalism becomes a tricky problem compared to the unconstrained motion tasks. In this case, the primary task is defined by the velocity tracking along the tangent space and force tracking along the normal space [46]. It should be noticed that only a small number of publications and research studies have addressed the problems of force-motion control and redundancy resolution within the same control framework. In particular, the force control scheme proposed in [55], handles the force tracking task as the secondary objective while the motion of the end effector is defined as the primary objective task. Moreover, the aforementioned method needs reseting when the contact is lost. In a recent work [46], a model free control scheme was proposed for a UVMS in rigid contact with the environment while simultaneously dealing with the aforementioned issues. More specifically, by means of a priority based inverse kinematic formulation proposed in [47], the UVMS operational limitations along with the contact maintenance task are formulated as inequality constraints within a cascade of quadratic programming problems. However, beyond the known implementation drawbacks of sliding mode control technique (chattering and high gain excitation) that is adopted at the low level, it requires the accurate 
knowledge of the bounds of the fluid disturbances. Moreover, the transient and steady state performance of the system is not considered at all.

In this work 1 , we propose a force-motion control strategy for a UVMS in compliant contact with a planar surface. The purpose of the controller is to simultaneously track a desired trajectory along the planar surface and a force along the normal direction. The proposed method does not require any knowledge er the UVMS dynamic parameters, or the stiffness model, or the disturbance profile. Furthermore, various performance issues such as: i) maintaining the contact, ii) tracking the desired trajectory, iii) inferior overshooting of the interaction force error and iv) robust steady state response are achieved. More, the novel formulation of the problem, allows the tracking of the desired force and position trajectories to be considered equally prioritized, while it enables us to treat various other operational limitations (e.g., joint limits) as secondary tasks, and thus fully decouple them. In particular, the proposed constrategy tackles all aforementioned challenges and further guarantees predefined behavior in terms of overshoot, convergence rate and maximum steady state error. Furthermore, the robustness of the proposed control strategy with respect to external disturbances is enhanced. Finally, the complexity of the proposed control law is significantly low. It is actually a static scheme involving only a few calculations to derive the control signal, which enables its onboard implementation straightforwardly.

The rest of the manuscript is organized as follows: In Section-2, the problem is rigorously formulated. The analytic description of the proposed method along with the corresponding stability proof are presented in Section-3. The efficiency of the proposed approach is illustrated and validated via simu-200 lated and experimental results in Section-4. Finally, Section-5

\section{Problem Formulation}

Consider an $n$ degrees of freedom UVMS in compliant contact with a planar surface. Let $\boldsymbol{q}=\left[\boldsymbol{q}_{a}^{\top}, \boldsymbol{q}_{m}^{\top}\right]^{\top} \in \mathbb{R}^{n}$ be the state variables of the UVMS, where $\boldsymbol{q}_{a}=\left[\boldsymbol{\eta}_{1}^{\top}, \boldsymbol{\eta}_{\mathbf{2}}^{\top}\right]^{\top} \in \mathbb{R}^{6}$ involves the position vector $\boldsymbol{\eta}_{1}=\left[x_{v}, y_{v}, z_{v}\right]^{\top}$ and the orientation $\boldsymbol{\eta}_{2}=[\phi, \theta, \psi]^{\top}$ of the vehicle expressed in the Eulerangles representation with respect to (w.r.t) an inertial frame $\{I\}$ and $\boldsymbol{q}_{m} \in \mathbb{R}^{n-6}$ is the angle vector of the manipulator's joints. Consider also the frame $\{E\}$ attached at the end-effector of the UVMS described by a position vector $\boldsymbol{x}_{e}=\left[x_{e}, y_{e}, z_{e}\right]^{\top} \in \mathbb{R}^{3}$ and a rotation matrix $\boldsymbol{R}_{e}=\left[\boldsymbol{n}_{e}, \boldsymbol{o}_{e}, \boldsymbol{\alpha}_{e}\right]$ w.r.t the inertial frame $\{I\}$. Let also $\omega_{e}$ be the rotational velocity of the end-effector

${ }^{1}$ Preliminary results of this work were presented in the 20th World Congress of the International Federation of Automatic Control (IFAC) 2017 [56]. In the current version, analytic results on force/position/orientation tracking control with guaranteed contact maintenance and experimental results are provided in addition.

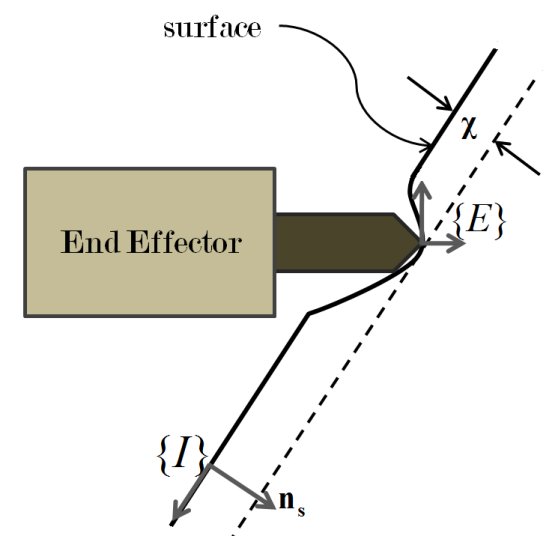

Figure 1: A graphical illustration of the UVMS end-effector in compliant contact with a planar surface.

that satisfies $S\left(\omega_{e}\right)=\dot{\boldsymbol{R}}_{e} \boldsymbol{R}_{e}^{\top}$, where

$$
\boldsymbol{S}(\boldsymbol{d})=\left[\begin{array}{ccc}
0 & -d_{z} & d_{y} \\
d_{z} & 0 & -d_{x} \\
-d_{y} & d_{x} & 0
\end{array}\right]
$$

is the skew-symmetric matrix of the vector $\boldsymbol{d}=\left[d_{x} d_{y} d_{z}\right]^{\top}$. Let also $\dot{\boldsymbol{x}}=\left[\dot{\boldsymbol{x}}_{e}^{\top}, \boldsymbol{\omega}_{e}^{\top}\right]^{\top} \in \mathbb{R}^{6}$ denote the velocity of the end-effector frame. Without loss of generality, we have [4]:

$$
\dot{\boldsymbol{x}}=\boldsymbol{J}(\boldsymbol{q}) \zeta
$$

where $\boldsymbol{\zeta}=\left[\boldsymbol{v}^{\top}, \dot{\boldsymbol{q}}_{m, i}^{\top}\right]^{\top} \in \mathbb{R}^{n}$ is the overall velocity vector that involves the body velocities of the vehicle $v$ and the joint velocities of the manipulator $\dot{\boldsymbol{q}}_{m, i}, i \in\{1, \ldots, n-6\}$ and $\boldsymbol{J}(\boldsymbol{q}) \in \mathbb{R}^{6 \times n}$ is the geometric Jacobian Matrix [4].

We assume that the UVMS has initially established contact with a planar surface, whose normal and tangential vectors expressed w.r.t the inertial frame $\{I\}$ are known. Thus, for the sake of simplicity, we consider the inertial frame $\{I\}$ attached at some point on the surface with its $x$-axis normal to the surface pointing inwards (See Fig.1). Now, let us denote the unit vector normal to the contact surface and the generalized normal vector as $\boldsymbol{n}_{s}=\left[\begin{array}{lll}1 & 0 & 0\end{array}\right]^{\top} \in \mathbb{R}^{3}$ and $\boldsymbol{n}=\left[\begin{array}{ll}\boldsymbol{n}_{s}^{\top} & \mathbf{0}_{3}^{\top}\end{array}\right]^{\top} \in \mathbb{R}^{6}$ respectively. We also assume that the end-effector is rigid, thus the contact compliance arises from the planar surface ${ }^{2}$. Hence, the deformation $\chi$ is given as a function of $\boldsymbol{x}_{e}$ as follows:

$$
\chi=\boldsymbol{n}_{s}^{\top} \boldsymbol{x}_{e}=x_{e}
$$

and its derivative is calculated by:

$$
\dot{\chi}=\boldsymbol{n}_{s}^{\top} \dot{\boldsymbol{x}}_{e}=\dot{x}_{e}
$$

During an intervention task, the UVMS exerts an interaction wrench $\lambda \in \mathbb{R}^{6}$ at the contact, which can be measured by a force/torque sensor attached to its end-effector. This interaction

\footnotetext{
${ }^{2}$ In case of UVMS with soft tip, the compliance may arise either from the tip side or the surface or both. Thus, the deformation can also be derived without affecting the subsequent analysis.
} 
wrench can be decomposed into: (i) $\boldsymbol{n} \boldsymbol{n}^{\top} \boldsymbol{\lambda}$ that is normal to the surface and (ii) $\left(\boldsymbol{I}_{6 \times 6}-\boldsymbol{n} \boldsymbol{n}^{\top}\right) \boldsymbol{\lambda}$ involving tangential forces and torques, owing to tangential deformations and friction terms. In this work, we assume that the normal force magnitude $f=\boldsymbol{n}^{\top} \boldsymbol{\lambda}$ is a positive and continuously differentiable nonlinear function of the material deformation $\chi$ :

$$
f=\Phi(\chi), \quad \forall \chi \geq 0 .
$$

The aforementioned general formulation includes several force deformation models such as the Hertz model [57] $(\Phi(\chi)=$ $k \chi^{\frac{3}{2}}, k>0$ ) or the quadratic model $\Phi(\chi)=k \chi^{2}, k>0$ [58]. The time derivative of the normal force magnitude in view of (3) is then given by:

$$
\dot{f}=\partial \Phi(\chi) \dot{x}_{e}
$$

where $\partial \Phi(\chi)=\frac{d \Phi}{d \chi}$ is strictly positive for all $\chi \geq \chi^{*}>0$, where $\chi^{*}$ is any strictly real positive number. Thus, there is an unknown strictly positive constant $\partial \Phi^{*}$ such that:

$$
\partial \Phi(\chi) \geq \partial \Phi^{*}>0, \forall \chi \geq \underline{\chi}^{*} .
$$

Without loss of generality, the dynamics of the UVMS in complaint contact with the environment can be formulated as [4]:

$$
M(q) \dot{\zeta}+C(q, \zeta) \zeta+D(q, \zeta) \zeta+g(q)+J^{\top}(q) \lambda+\delta(q, \zeta, t)=\tau
$$

where $\boldsymbol{\delta}(\boldsymbol{q}, \boldsymbol{\zeta}, t)$ encapsulates bounded unmodeled terms and external disturbances (sea waves and currents). Moreover, $\tau \in \mathbb{R}^{n}$ denotes the control input at the joint/thruster level, $\boldsymbol{M}(\boldsymbol{q})$ is the positive definite inertial matrix, $\boldsymbol{C}(\boldsymbol{q}, \boldsymbol{\zeta})$ represents coriolis and centrifugal terms, $\boldsymbol{D}(\boldsymbol{q}, \boldsymbol{\zeta})$ models dissipative effects, $\boldsymbol{g}(\boldsymbol{q})$ encapsulates the gravity and buoyancy effects and $\boldsymbol{J}^{\top}(\boldsymbol{q}) \boldsymbol{\lambda}$ represents the effect of the external forces/torques applied at the end-effector owing to the contact.

Finally, the problem to be solved in this work is formulated as follows:

Problem 1. Given a UVMS, a desired force profile along the normal to the surface direction $f^{d}(t)$, a desired position trajectory $\left[y_{e}^{d}(t), z_{e}^{d}(t)\right]^{\top} \in \mathbb{R}^{2}$ on the planar surface, as well as a smooth rotation matrix target $\boldsymbol{R}^{d}(t)=\left[\boldsymbol{n}^{d}(t), \boldsymbol{o}^{d}(t), \boldsymbol{\alpha}^{d}(t)\right]$, design a feedback control law, without incorporating any information regarding either the UVMS dynamics or the force deformation model, such that the following are satisfied:

1. Predefined behavior in terms of overshoot, convergence rate and maximum steady state error on the force/position/orientation tracking errors;

2. Contact maintenance;

3. Bounded closed loop signals;

4. Robustness against external disturbances.

\section{Control Methodology}

Typically, in a force/position control problem the robot endeffector should track a force trajectory along the normal to the surface direction, a desired position trajectory on the surface and possibly attain a desired orientation related to the contact surface. Hence, we define the force, position, orientation and the overall errors as:

$$
\begin{aligned}
e_{f} & =f-f^{d} \in \mathbb{R}, \\
\boldsymbol{e}_{p} & \triangleq\left[\begin{array}{l}
e_{y} \\
e_{z}
\end{array}\right]=\left[\begin{array}{l}
y_{e}-y_{e}^{d} \\
z_{e}-z_{e}^{d}
\end{array}\right] \in \mathbb{R}^{2}, \\
\boldsymbol{e}_{o} & \triangleq\left[\begin{array}{l}
e_{o_{1}} \\
e_{o_{2}} \\
e_{o_{3}}
\end{array}\right]=\frac{1}{2}\left(\boldsymbol{n}_{e} \times \boldsymbol{n}^{d}+\boldsymbol{o}_{e} \times \boldsymbol{o}^{d}+\boldsymbol{\alpha}_{e} \times \boldsymbol{\alpha}^{d}\right) \in \mathbb{R}^{3}, \\
\boldsymbol{e} & \triangleq\left[e_{f}, e_{y}, e_{z}, e_{o_{1}}, e_{o_{2}}, e_{o_{3}}\right]^{\top} \in \mathbb{R}^{6}
\end{aligned}
$$

Notice that for the orientation error $\boldsymbol{e}_{o}$, we have employed the outer product formulation [59, 60] of the end-effector rotation matrix $\boldsymbol{R}_{e}$ and the desired rotation matrix $\boldsymbol{R}^{d}$ to relax the representation singularity issue that is inherent in case Euler angles are adopted ${ }^{3}$. Differentiating $(8 \mathrm{a})-(8 \mathrm{c})$ with respect to time and in view of (5), we obtain:

$$
\begin{aligned}
& \dot{e}_{f}=\partial f(\chi) \dot{x}_{e}-\dot{f}^{d}, \\
& \dot{\boldsymbol{e}}_{p}=\left[\begin{array}{l}
\dot{e}_{y} \\
\dot{e}_{z}
\end{array}\right]=\left[\begin{array}{l}
\dot{y}_{e}-\dot{y}_{e}^{d} \\
\dot{z}_{e}-\dot{z}_{e}^{d}
\end{array}\right], \\
& \dot{\boldsymbol{e}}_{o}=\left[\begin{array}{l}
\dot{e}_{O_{1}} \\
\dot{e}_{o_{2}} \\
\dot{e}_{o_{3}}
\end{array}\right]=\boldsymbol{L} \boldsymbol{\omega}_{e}-\boldsymbol{L} \boldsymbol{\omega}^{d},
\end{aligned}
$$

where $\boldsymbol{L}$ is defined as:

$$
\boldsymbol{L}=\frac{1}{2}\left[\boldsymbol{S}\left(\boldsymbol{n}_{e}\right) \boldsymbol{S}\left(\boldsymbol{n}^{d}\right)+\boldsymbol{S}\left(\boldsymbol{o}_{e}\right) \boldsymbol{S}\left(\boldsymbol{o}^{d}\right)+\boldsymbol{S}\left(\boldsymbol{\alpha}_{e}\right) \boldsymbol{S}\left(\boldsymbol{\alpha}^{d}\right)\right]
$$

which is full rank when the relative orientation between the frames $\boldsymbol{R}_{e}$ and $\boldsymbol{R}^{d}$ is confined less than $90^{\circ}$ for an angle-axis local parametrization and hence is not restrictive for practical cases [60].

\subsection{Control Design}

An appropriate methodology to meet the control objectives of this work is the prescribed performance control technique [61, 62, 63], which is adapted here in order to achieve predefined transient and steady state response bounds for the errors. By prescribed performance control we mean that the force, position and orientation errors evolve strictly within a predefined region that is bounded by decaying functions of time, which is mathematically expressed as:

$$
-\underline{M}_{i} \rho_{i}(t)<e_{i}(t)<\bar{M}_{i} \rho_{i}(t), i \in\left\{f, y, z, o_{1}, o_{2}, o_{3}\right\}, \forall t \geq 0
$$

with

$$
\begin{aligned}
\rho_{i}(t)=\left(1-\frac{\rho_{i}^{\infty}}{\max \left\{\underline{M}_{i}, \bar{M}_{i}\right\}}\right) \exp \left(-l_{i} t\right)+ & \left(\frac{\rho_{i}^{\infty}}{\max \left\{\underline{M}_{i}, \bar{M}_{i}\right\}}\right), \\
& i \in\left\{f, y, z, o_{1}, o_{2}, o_{3}\right\} .
\end{aligned}
$$

\footnotetext{
${ }^{3}$ Other representations, such as unit quaternions, can also be considered in order to describe the orientation errors. For more details the reader is referred to [59].
} 
The constants $M_{i}, \bar{M}_{i}$, are selected such that (11) is satisfied at $t=0$ (i.e., $\left.-\underline{M}_{i}<e_{i}(0)<\bar{M}_{i}\right)$. The constant $\rho_{i}^{\infty}=\lim _{t \rightarrow \infty} \rho_{i}(t)$ represents the maximum allowable size of $e_{i}(t)$ at the steady state, which can be set arbitrarily small to a value reflecting the resolution of the measurement device, thus achieving practical convergence of $e_{i}(t)$ to zero. Furthermore, the decreasing rate of $\rho_{i}(t)$, which is affected by the constant $l_{i}$ introduces a lower bound of the required speed of convergence of $e_{i}(t)$, while the maximum overshoot is prescribed less than $\underline{M}_{i} \rho_{i}(0)$ or $\bar{M}_{i} \rho_{i}(0)$. Thus, the appropriate selection of the performance functions $\rho_{i}(t), i \in\left\{f, y, z, o_{1}, o_{2}, o_{3}\right\}$ as well as of the design constants $\underline{M}_{i}, \bar{M}_{i}, i \in\left\{f, y, z, o_{1}, o_{2}, o_{3}\right\}$ encapsulates perfor- ${ }_{270}$ mance characteristics for the corresponding tracking errors $e_{i}$, $i \in\left\{f, y, z, o_{1}, o_{2}, o_{3}\right\}$.

In particular, meeting the performance bounds for the force error, as described in (11), allows us further to guarantee a priori that contact with the surface is never lost (i.e., $f(t) \geq f^{*}>$ $0, \forall t \geq 0$ for a positive constant $\left.f^{*}\right)$ and excessive interaction forces are avoided (i.e, $f(t) \leq \overline{\bar{f}}^{*}>0, \forall t \geq 0$ for a positive constant $\left.\bar{f}^{*}>f^{*}>0\right)$. In this spirit, $\underline{M}_{f}, \bar{M}_{f}$ and $\rho_{f}(t)$ are selected to further satisfy: $\inf _{t \geq 0}\left\{-\underline{M}_{f} \rho_{f}(t)+f^{d}(t)\right\}>f^{*}$, $\sup _{t \geq 0}\left\{\bar{M}_{f} \rho_{f}(t)+f^{d}(t)\right\}<\bar{f}^{*}$. Hence, owing to (12) it can be verified that the satisfaction of the performance bounds for the force error guarantees further that $0<\inf _{t \geq 0}\left\{-\underline{M}_{f} \rho_{f}(t)+\right.$ $\left.f^{d}(t)\right\} \leq f(t) \leq \sup _{t>0}\left\{\bar{M}_{f} \rho_{f}(t)+f^{d}(t)\right\}, \forall t \geq 0$. Finally, in $\partial f(\chi) \leq \overline{\partial f}$.

In the sequel, we propose a state feedback control protocol that does not incorporate any information regarding the UVMS dynamic model (7) or the deformation model and achieves force/position/orientation tracking of the corresponding smooth and bounded desired trajectories with prescribed transient and steady state response. The overall control architecture is illustrated in Fig2

Level I-a: Select the performance functions $\rho_{i}(t)$ and the corresponding positive parameters $\underline{M}_{i}, \bar{M}_{i}$ such that $-\underline{M}_{i}<e_{i}(0)<$ $\bar{M}_{i}, \forall i \in\left\{f, y, z, o_{1}, o_{2}, o_{3}\right\}$ and the desired transient and steady state performance specifications are properly encapsulated.

Level I-b: Define the transformed errors $\varepsilon_{i}$ as:

$$
\varepsilon_{i}\left(\xi_{i}\right)=\ln \left(\frac{1+\frac{\xi_{i}}{\underline{\underline{M}}_{i}}}{1-\frac{\xi_{i}}{\overline{M_{i}}}}\right), i \in\left\{f, y, z, o_{1}, o_{2}, o_{3}\right\}
$$

where

$$
\xi_{i}(t)=\frac{e_{i}(t)}{\rho_{i}(t)}, i \in\left\{f, y, z, o_{1}, o_{2}, o_{3}\right\},
$$

denote the normalized force/position/orientation errors and se-275 lect the end-effector reference velocity:

$$
\dot{\boldsymbol{x}}^{r}\left(\boldsymbol{\xi}_{x}, t\right)=-\boldsymbol{K} \mathbf{P}^{-1}(t) \operatorname{diag}\left[\boldsymbol{I}_{3 \times 3}, \boldsymbol{L}^{-1}\right] \boldsymbol{R}\left(\boldsymbol{\xi}_{x}\right) \boldsymbol{\varepsilon}\left(\boldsymbol{\xi}_{x}\right)
$$

where

$$
\boldsymbol{R}\left(\boldsymbol{\xi}_{x}\right)=\underset{i \in\left\{f, y, z, 0_{1}, o_{2}, o_{3}\right\}}{\operatorname{diag}}\left[\frac{\frac{1}{\underline{\underline{M}}_{i}}+\frac{1}{\bar{M}_{i}}}{\left(1-\frac{\xi_{i}}{\underline{\underline{M}}_{i}}\right)\left(1+\frac{\xi_{i}}{\bar{M}_{i}}\right)}\right],
$$

$$
\boldsymbol{\varepsilon}\left(\boldsymbol{\xi}_{x}\right) \triangleq\left[\varepsilon_{f}\left(\xi_{f}\right), \varepsilon_{y}\left(\xi_{y}\right), \varepsilon_{z}\left(\xi_{z}\right), \varepsilon_{o_{1}}\left(\xi_{o_{1}}\right), \varepsilon_{o_{2}}\left(\xi_{o_{2}}\right), \varepsilon_{o_{3}}\left(\xi_{o_{3}}\right)\right]^{\top},
$$

with $\boldsymbol{\xi}_{x}=\left[\xi_{f}, \xi_{y}, \xi_{z}, \xi_{o_{1}}, \xi_{o_{2}}, \xi_{o_{3}}\right]^{\top}, \mathbf{P}(t) \triangleq \underset{i \in\left\{f, y, z, o_{1}, o_{2}, o_{3}\right\}}{\operatorname{diag}}\left[\rho_{i}(t)\right]$ and $\boldsymbol{K}=\underset{i \in\left\{f, y, z, O_{1}, o_{2}, o_{3}\right\}}{\operatorname{diag}}\left[k_{i}\right]$ is a positive diagonal gain matrix. Subsequently, the task-space desired motion profile $\dot{\boldsymbol{x}}^{r}$ can be expressed equivalently in the configuration space via:

$$
\boldsymbol{\zeta}^{r}(t)=\boldsymbol{J}(\boldsymbol{q})^{\#} \dot{\boldsymbol{x}}^{r}+\left(\boldsymbol{I}_{n \times n}-\boldsymbol{J}(\boldsymbol{q})^{\#} \boldsymbol{J}(\boldsymbol{q})\right) \dot{\boldsymbol{x}}^{0} \in \mathbb{R}^{n}
$$

where $\boldsymbol{J}(\boldsymbol{q})^{\#}$ denotes the generalized pseudo-inverse [64] of the Jacobian $\boldsymbol{J}(\boldsymbol{q})$ and $\dot{\boldsymbol{x}}^{0}$ denotes secondary tasks (e.g., maintaining manipulator's joint limits, increasing manipulability) to be regulated independently since they do not contribute to the endeffector's velocity [48] (i.e., they belong to the null space of the Jacobian $\boldsymbol{J}(\boldsymbol{q}){ }^{4}$

Level II-a: Define the velocity error vector as:

$$
\boldsymbol{e}_{\zeta}(t) \triangleq\left[e_{\zeta_{1}}(t), \ldots, e_{\zeta_{n}}(t)\right]^{\top}=\zeta(t)-\zeta^{r}(t) \in \mathbb{R}^{n}
$$

and select the corresponding performance functions:

$$
\rho_{\zeta_{i}}(t)=\left(\rho_{\zeta_{i}}^{0}-\rho_{\zeta_{i}}^{\infty}\right) \exp \left(-l_{\zeta_{i}} t\right)+\rho_{\zeta_{i}}^{\infty}, i=1, \ldots, n
$$

with $\rho_{\zeta_{i}}^{0}>\left|e_{\zeta_{i}}(0)\right|, \rho_{\zeta_{i}}^{\infty}>0$ and $l_{\zeta_{i}}>0, i=1, \ldots n$. Notice that similarly to the force/position/orientation errors we intend to enforce transient and steady state response on the velocity errors $e_{\zeta_{i}}(t), i=1, \ldots, n$ as well by satisfying:

$$
-\rho_{\zeta_{i}}(t)<e_{\zeta_{i}}(t)<\rho_{\zeta_{i}}(t), \forall t \geq 0 i=1, \ldots, n
$$

Level II-b: Define the transformed velocity error vector:

$\boldsymbol{\varepsilon}_{\zeta}\left(\boldsymbol{\xi}_{\zeta}\right) \triangleq\left[\varepsilon_{\zeta_{1}}\left(\xi_{\zeta_{1}}\right), \ldots, \varepsilon_{\zeta_{n}}\left(\xi_{\zeta_{n}}\right)\right]^{\top}=\left[\ln \left(\frac{1+\xi_{\zeta_{1}}}{1-\xi_{\zeta_{1}}}\right), \ldots, \ln \left(\frac{1+\xi_{\zeta_{n}}}{1-\xi_{\zeta_{n}}}\right)\right]^{\top}$

where

$$
\boldsymbol{\xi}_{\zeta}(t) \triangleq\left[\xi_{\zeta_{1}}, \ldots, \xi_{\zeta_{n}}\right]^{\top}=\mathbf{P}_{\zeta}^{-1}(t) \boldsymbol{e}_{\zeta}(t)
$$

denotes the normalized velocity error vector, with $\mathbf{P}_{\zeta}(t)=$ diag $\left[\rho_{\zeta_{i}}(t)\right]$ and design the state feedback control law: $i=1, \ldots, n$

$$
\boldsymbol{\tau}\left(\boldsymbol{e}_{\zeta}(t), t\right)=-\boldsymbol{K}_{\zeta} \mathbf{P}_{\zeta}^{-1}(t) \boldsymbol{R}_{\zeta}\left(\boldsymbol{\xi}_{\zeta}\right) \boldsymbol{\varepsilon}_{\zeta}\left(\boldsymbol{\xi}_{\zeta}\right)
$$

where

$$
\boldsymbol{R}_{\zeta}\left(\boldsymbol{\xi}_{\zeta}\right)=\underset{i=1, \ldots, n}{\operatorname{diag}}\left[\frac{2}{1-\xi_{\zeta_{i}}^{2}}\right]
$$

and $\boldsymbol{K}_{\zeta}>0$ is a diagonal gain matrix.

Theorem 1. The proposed state feedback control law (13)-25] enforces tracking of: $i)$ the desired normal force trajectory $f^{d}(t)$, ii) the desired position trajectory $\left[y_{e}^{d}(t), z_{e}^{d}(t)\right]^{\top} \in \mathbb{R}^{2}$ on the planar surface as well as iii) the smooth rotation matrix target $\boldsymbol{R}^{d}(t)=\left[\boldsymbol{n}^{d}(t), \boldsymbol{o}^{d}(t), \boldsymbol{\alpha}^{d}(t)\right]$ with the desired transient and steady state performance.

\footnotetext{
${ }^{4}$ For more details on task priority based control and redundancy resolution for UVMSs the reader is referred to [48] and [54].
} 


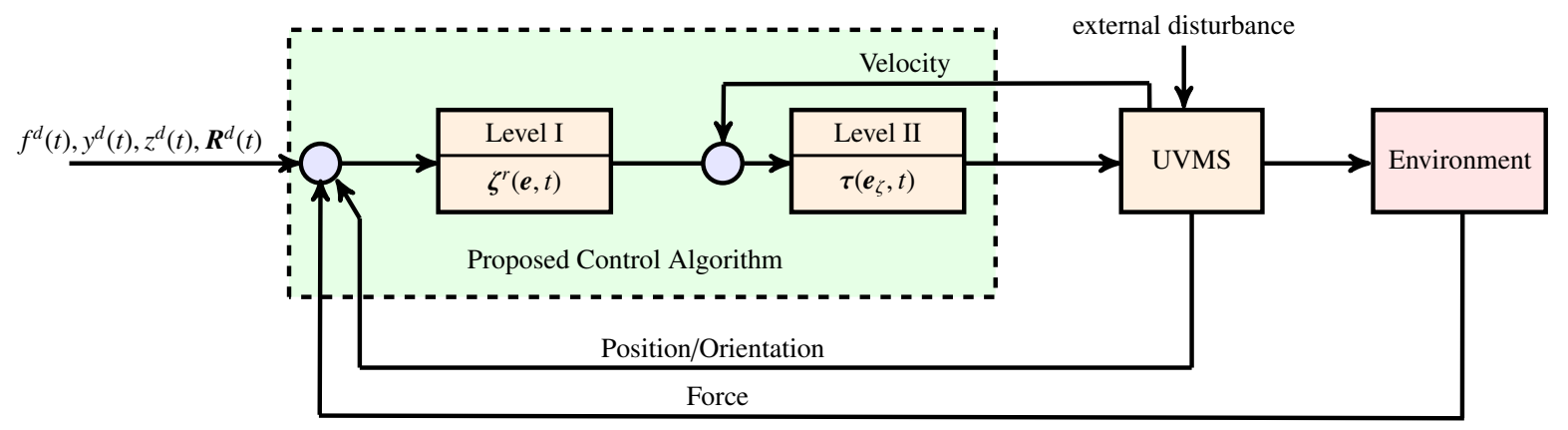

Figure 2: The closed loop block diagram of the proposed control scheme.

Proof. First, let us define the overall normalized error vector $\boldsymbol{\xi}=\left[\boldsymbol{\xi}_{x}^{\top}, \boldsymbol{\xi}_{\zeta}^{\top}\right]^{\top}$. Differentiating (14) and [23) with respect to time and substituting the system dynamics (7), (9), (15), 19) and (24), we obtain the closed loop system dynamics:

$$
\begin{aligned}
\dot{\boldsymbol{\xi}}_{x} \triangleq & h_{x}\left(\boldsymbol{\xi}_{x}, t\right) \\
= & -\mathbf{P}^{-1}(t) \operatorname{diag}\left[\partial \Phi(\chi), \boldsymbol{I}_{5 \times 5}\right] \boldsymbol{K} \mathbf{P}^{-1}(t) \boldsymbol{R}\left(\boldsymbol{\xi}_{x}\right) \boldsymbol{\varepsilon}\left(\boldsymbol{\xi}_{x}\right) \\
& +\mathbf{P}^{-1}(t)\left[\operatorname{diag}\left[\partial \Phi(\chi), \boldsymbol{I}_{2 \times 2}, \boldsymbol{L}\right] \boldsymbol{J} \mathbf{P}_{\zeta}(t) \boldsymbol{\xi}_{\zeta}\right. \\
& \left.-\left[\dot{f}^{d}, \dot{y}^{d}, \dot{z}^{d},\left[\boldsymbol{L} \boldsymbol{\omega}^{d}\right]^{\top}\right]^{\top}-\dot{\mathbf{P}}(t) \boldsymbol{\xi}_{x}\right]
\end{aligned}
$$

$$
\begin{aligned}
\dot{\boldsymbol{\xi}}_{\zeta}\left(\xi_{\zeta}, t\right) & \triangleq h_{\zeta}\left(\boldsymbol{\xi}_{\zeta}, t\right) \\
& =-\mathbf{P}_{\zeta}^{-1}(t) \boldsymbol{M}^{-1} \boldsymbol{K}_{\zeta} \mathbf{P}_{\zeta}^{-1}(t) \boldsymbol{R}_{\zeta}\left(\boldsymbol{\xi}_{\zeta}\right) \boldsymbol{\varepsilon}_{\zeta}\left(\boldsymbol{\xi}_{\zeta}\right)- \\
& -\mathbf{P}_{\zeta}^{-1}(t)\left[\boldsymbol { M } ^ { - 1 } \left(\boldsymbol{C} \cdot\left(\mathbf{P}_{\zeta}(t) \boldsymbol{\xi}_{\zeta}+\zeta^{r}\right)+\boldsymbol{D} \cdot\left(\mathbf{P}_{\zeta}(t) \boldsymbol{\xi}_{\zeta}+\boldsymbol{\zeta}^{r}\right)\right.\right. \\
& \left.\left.+\boldsymbol{g}+\boldsymbol{J}^{\top} \boldsymbol{\lambda}+\boldsymbol{\delta}(t)\right)+\dot{\mathbf{P}}_{\zeta}(t) \boldsymbol{\xi}_{\zeta}+\dot{\zeta}^{r}\right]
\end{aligned}
$$

which can be written in compact form as:

$$
\dot{\boldsymbol{\xi}} \triangleq h(\boldsymbol{\xi}, t)=\left[h_{x}^{\top}\left(\boldsymbol{\xi}_{\boldsymbol{x}}, t\right), h_{\zeta}^{\top}\left(\boldsymbol{\xi}_{\zeta}, t\right)\right]^{\top}
$$

Let us define the open set $\Omega_{\xi}=\Omega_{\xi_{x}} \times \Omega_{\xi_{\zeta}}$ with $\Omega_{\xi_{x}} \triangleq\left(-\underline{M}_{f}, \bar{M}_{f}\right) \times\left(-\underline{M}_{y}, \bar{M}_{y}\right) \times\left(-\underline{M}_{z}, \bar{M}_{z}\right) \times\left(-\underline{M}_{o_{1}}, \bar{M}_{o_{1}}\right) \times$ $\left(-\underline{M}_{o_{2}}, \bar{M}_{o_{2}}\right) \times\left(-\underline{M}_{o_{3}}, \bar{M}_{o_{3}}\right)$ and $\Omega_{\xi_{\zeta}} \triangleq(-1,1)^{n}$. In what follows, we proceed in two phases. First we ensure the existence of a unique maximal solution $\xi(t)$ of $(28)$ over the set $\Omega_{\xi}$ for a time interval $\left[0, t_{\max }\right)$ (i.e., $\boldsymbol{\xi}(t) \in \Omega_{\xi}, \forall t \in\left[0, t_{\max }\right)$ ). Then, we prove that the proposed controller guarantees, for all $t \in\left[0, t_{\max }\right)$ the boundedness of all closed loop signals as well as that $\boldsymbol{\xi}(t)$ remains strictly within the set $\Omega_{\xi}$, which leads by contradiction to $t_{\max }=\infty$ and consequently to the satisfaction of (11) and (21), thus completing the proof.

Phase A: The set $\Omega_{\xi}$ is nonempty and open. Moreover (11) and (21) leads to $-\underline{M}_{i}<\xi_{i}(0)<\bar{M}_{i}, i \in\left\{f, y, z, o_{1}, o_{2}, o_{3}\right\}$ and $-1<\xi_{\zeta_{i}}(0)<\overline{1}, i=1, \ldots, n$. Thus, we guarantee that $\boldsymbol{\xi}_{x}(0) \in \Omega_{\xi_{x}}$ and $\boldsymbol{\xi}_{\zeta}(0) \in \Omega_{\xi_{\zeta}}$. Additionally, $h(\xi, t)$, as defined in (28), is continuous on $t$ and locally Lipschitz on $\boldsymbol{\xi}$ over $\Omega_{\xi}$. Therefore, the hypotheses of the Theorem 54 in [65] (pp. 476) hold and the existence of a maximal solution $\boldsymbol{\xi}(t)$ of $(28)$ on a time interval $\left[0, t_{\max }\right)$ such that $\boldsymbol{\xi}(t) \in \Omega_{\xi}, \forall t \in\left[0, t_{\max }\right)$ is ensured.

Phase B: In Phase A, we proved that $\boldsymbol{\xi}(t) \in \Omega_{\xi}, \forall t \in\left[0, t_{\max }\right)$, thus it can be concluded that:

$$
\begin{gathered}
\xi_{i}(t)=\frac{e_{i}(t)}{\rho_{i}(t)} \in\left(-\underline{M}_{i}, \bar{M}_{i}\right), i \in\left\{f, y, z, o_{1}, o_{2}, o_{3}\right\} \\
\xi_{\zeta_{i}}(t)=\frac{e_{\zeta_{i}}}{\rho_{\zeta_{i}}} \in(-1,1), i=1, \ldots, n
\end{gathered}
$$

for all $t \in\left[0, t_{\max }\right)$, from which we obtain that $e_{i}(t)$ and $e_{\zeta_{i}}(t)$ are lower and upper bounded by $-\underline{M}_{i} \rho_{i}(t), \bar{M}_{i} \rho_{i}(t)$ and $-\rho_{\zeta_{i}}(t), \rho_{\zeta_{i}}(t)$, respectively. Therefore, the transformed error vectors $\varepsilon_{i}\left(\xi_{i}\right)$ and $\varepsilon_{\zeta_{i}}\left(\xi_{\zeta_{i}}\right)$ designated in (13) and (22), respectively, are well-defined for all $t \in\left[0, t_{\max }\right)$. Hence, consider the positive definite and radially unbounded function:

$$
V(\boldsymbol{\varepsilon})=\frac{1}{2} \boldsymbol{\varepsilon}^{\top}\left(\boldsymbol{\xi}_{x}\right) \boldsymbol{\varepsilon}\left(\boldsymbol{\xi}_{x}\right)=\frac{1}{2} \varepsilon_{f}^{2}+\frac{1}{2}\left\|\boldsymbol{\varepsilon}_{p}\right\|^{2}+\frac{1}{2}\left\|\boldsymbol{\varepsilon}_{o}\right\|^{2}
$$

with $\boldsymbol{\varepsilon}_{p}=\left[\varepsilon_{p_{1}}, \varepsilon_{p_{2}}\right]^{\top}$ and $\boldsymbol{\varepsilon}_{o}=\left[\varepsilon_{o_{1}}, \varepsilon_{o_{2}}, \varepsilon_{o_{3}}\right]^{\top}$. Differentiating $V(\varepsilon)$ with respect to time and substituting $[26]$ results in:

$$
\begin{aligned}
\dot{V}= & -\boldsymbol{\varepsilon}^{\top}\left(\boldsymbol{\xi}_{x}\right) \boldsymbol{R}\left(\boldsymbol{\xi}_{x}\right) \mathbf{P}^{-1}(t) \operatorname{diag}\left[\partial \Phi(\chi), \boldsymbol{I}_{5 \times 5}\right] \boldsymbol{K} \mathbf{P}^{-1}(t) \boldsymbol{R}\left(\boldsymbol{\xi}_{x}\right) \boldsymbol{\varepsilon}^{\top}\left(\boldsymbol{\xi}_{x}\right) \\
& +\boldsymbol{\varepsilon}^{\top}\left(\boldsymbol{\xi}_{x}\right) \boldsymbol{R}\left(\boldsymbol{\xi}_{x}\right) \mathbf{P}^{-1}(t) \boldsymbol{B}_{x}
\end{aligned}
$$

where

$$
\boldsymbol{B}_{x}=\operatorname{diag}\left[\partial \Phi(\chi), \boldsymbol{I}_{2 \times 2}, \boldsymbol{L}\right] \boldsymbol{J} \mathbf{P}_{\zeta}(t) \boldsymbol{\xi}_{\zeta}-\left[\dot{f}^{d}, \dot{y}^{d}, \dot{z}^{d},\left[\boldsymbol{L} \boldsymbol{\omega}^{d}\right]^{\top}\right]^{\top}-\dot{\mathbf{P}}(t) \boldsymbol{\xi}_{x}
$$

It is well known that the Jacobian $\boldsymbol{J}$ is bounded by definition. Moreover, since, $\dot{f}^{d}(t), \dot{y}^{d}(t), \dot{z}^{d}(t), w^{d}(t), \rho_{i}(t) i \in$ $\left\{f, y, z, o_{1}, o_{2}, o_{3}\right\}$ and $\rho_{\zeta_{i}}(t), i=1, \ldots, n$ are bounded by construction and $\xi_{i}, \xi_{\zeta_{i}}$ are also bounded within the compact sets $\Omega_{\xi_{x}}$ and $\Omega_{\xi_{\zeta}}$ owing to (29), we conclude the existence of a position constant $\bar{\varepsilon}$ such that:

$$
\left|\boldsymbol{\varepsilon}\left(\boldsymbol{\xi}_{x}(t)\right)\right| \leq \bar{\varepsilon}, \forall t \in\left[0, t_{\max }\right)
$$

Furthermore, from (13), and invoking the inverse logarithmic function, we obtain:

$-\underline{M}_{i}<\underline{\xi}_{i} \leq \xi_{i}(t) \leq \bar{\xi}_{i}<\bar{M}_{i}, \forall t \in\left[0, t_{\max }\right), i \in\left\{f, o_{1}, o_{2}, p_{1}, p_{2}, p_{3}\right\}$ 
where:

$$
\underline{\xi}_{i}=-\underline{M}_{i} \frac{\exp (\bar{\varepsilon})-1}{\exp (\bar{\varepsilon})+\frac{\underline{M}_{i}}{\bar{M}_{i}}}, \quad \bar{\xi}_{i}=\bar{M}_{i} \frac{\exp (\bar{\varepsilon})-1}{\exp (\bar{\varepsilon})+\frac{\bar{M}_{i}}{\underline{M}_{i}}}
$$

Owing to (34) and (18) it can concluded that the reference velocity vector $\zeta^{r}$ remains bounded for all $t \in\left[0, t_{\max }\right)$ as well. ${ }^{320}$ Moreover, invoking $\zeta=\zeta^{r}(t)+\mathbf{P}_{\zeta}(t) \boldsymbol{\xi}_{\zeta}$ from 23, we also conclude the boundedness of $\zeta$ for all $t \in\left[0, t_{\max }\right)$. Finally, differentiating $\zeta^{r}(t)$ w.r.t time and employing 26, 29) and (34), we conclude the boundedness of $\dot{\zeta}^{r}(t), \forall t \in\left[0, t_{\max }\right)$ too.

Now let us consider the positive definite and radially un- ${ }^{325}$ bounded function $V_{\zeta}\left(\boldsymbol{\varepsilon}_{\zeta}\right)=\frac{1}{2}\left\|\boldsymbol{\varepsilon}_{\zeta}\right\|^{2}$. Differentiating $V_{\zeta}$ with respect to time, substituting (27) and employing the continuity of $\boldsymbol{M}, \boldsymbol{C}, \boldsymbol{D}, \boldsymbol{g}, \boldsymbol{\lambda}, \boldsymbol{\delta}, \boldsymbol{\xi}_{x}, \boldsymbol{\xi}_{\zeta}, \dot{\mathbf{P}}_{\zeta}, \dot{\boldsymbol{\zeta}}^{r}, \forall t \in\left[0, t_{\max }\right)$, we obtain:

$$
\dot{V}_{\zeta} \leq\left\|\mathbf{P}_{\zeta}^{-1} \boldsymbol{R}_{\zeta}\left(\boldsymbol{\xi}_{\zeta}\right) \boldsymbol{\varepsilon}_{\zeta}\right\|\left(B_{\zeta}-\lambda_{M} \boldsymbol{K}_{\zeta}\left\|\mathbf{P}_{\zeta}^{-1} \boldsymbol{R}_{\zeta}\left(\boldsymbol{\xi}_{\zeta}\right) \boldsymbol{\varepsilon}_{\zeta}\right\|\right)
$$

$\forall t \in\left[0, t_{\max }\right)$, where $\lambda_{M}$ is the minimum eigenvalue of the positive definite matrix $\boldsymbol{M}^{-1}$ and $B_{\zeta}$ is a positive constant independent of $t_{\max }$, that satisfies:

$$
\begin{aligned}
B_{\zeta} \geq & \| \boldsymbol{M}^{-1}\left(\boldsymbol{C} \cdot\left(\mathbf{P}_{\zeta} \boldsymbol{\xi}_{\zeta}+\boldsymbol{\zeta}^{r}(t)\right)+\boldsymbol{D} \cdot\left(\mathbf{P}_{\zeta} \boldsymbol{\xi}_{\zeta}+\boldsymbol{\zeta}^{r}(t)\right)\right. \\
& \left.+\boldsymbol{g}+\boldsymbol{J}^{\top} \boldsymbol{\lambda}+\boldsymbol{\delta}(t)+\dot{\mathbf{P}}_{\zeta} \boldsymbol{\xi}_{\zeta}+\dot{\boldsymbol{\zeta}}^{r}\right) \|
\end{aligned}
$$

Thus, we conclude that:

$$
\left\|\boldsymbol{\varepsilon}_{\zeta}\left(\boldsymbol{\xi}_{\zeta}\right)\right\| \leq \bar{\varepsilon}_{\zeta}, \forall t \in\left[0, t_{\max }\right)
$$

Furthermore, from (25) and invoking $\left|\varepsilon_{\zeta_{i}}\right| \leq \bar{\varepsilon}_{\zeta}$, we obtain:

$$
-1<\underline{\xi}_{\zeta_{i}} \leq \xi_{\zeta_{i}}(t) \leq \bar{\xi}_{\zeta_{i}}<1, \forall t \in\left[0, t_{\max }\right), i=1, \ldots, n
$$

where

$$
\underline{\xi}_{\zeta_{i}}=\frac{-\exp \left(\bar{\varepsilon}_{\zeta}\right)-1}{-\exp \left(\bar{\varepsilon}_{\zeta}\right)+1}, \quad \bar{\xi}_{\zeta_{i}}=\frac{\exp \left(\bar{\varepsilon}_{\zeta}\right)-1}{\exp \left(\bar{\varepsilon}_{\zeta}\right)+1}
$$

which also leads to the boundedness of the control law (24) for all $t \in\left[0, t_{\max }\right)$.

Subsequently, we will show that $t_{\max }$ can be extended to in- ${ }^{350}$ finity. Obviously, notice by (34) and (37) that $\xi(t) \in \Omega_{\xi}^{\prime} \triangleq$ $\Omega_{\xi_{x}}^{\prime} \times \Omega_{\xi_{\zeta}}^{\prime}, \forall t \in\left[0, t_{\max }\right)$, where:

$\Omega_{\xi_{x}}^{\prime}=\left[\underline{\xi}_{f}, \bar{\xi}_{f}\right] \times\left[\underline{\xi}_{y}, \bar{\xi}_{y}\right] \times\left[\underline{\xi}_{z}, \bar{\xi}_{z}\right] \times\left[\underline{\xi}_{o_{1}}, \bar{\xi}_{o_{1}}\right] \times\left[\underline{\xi}_{o_{2}}, \bar{\xi}_{o_{2}}\right] \times\left[\underline{\xi}_{o_{3}}, \bar{\xi}_{o_{3}}\right]$ $\Omega_{\xi_{\zeta}}^{\prime}=\left[\underline{\xi}_{\zeta_{1}}, \bar{\xi}_{\zeta_{1}}\right] \times \ldots \times\left[\underline{\xi}_{\zeta_{n}}, \bar{\xi}_{\zeta_{n}}\right]$

are nonempty and compact subsets of $\Omega_{\xi_{x}}$ and $\Omega_{\xi_{\zeta}}$, respectively. Hence, assuming that $t_{\max }<\infty$ and since $\Omega_{\xi}^{\prime} \subset \Omega_{\xi}$, Proposition C.3.6 in [65] (pp. 481) dictates the existence of a time instant $t^{\prime} \in\left[0, t_{\max }\right)$ such that $\boldsymbol{\xi}\left(t^{\prime}\right) \notin \Omega_{\xi}^{\prime}$, which is a clear contradiction. Therefore, $t_{\max }=\infty$. Thus, all closed loop signals remain bounded and moreover $\boldsymbol{\xi}(t) \in \Omega_{\xi}^{\prime}, \forall t \geq 0$. Finally, from $(14)^{360}$ and (34) we conclude that:

$-\underline{M}_{i} \rho_{i}(t)<-\underline{M}_{i} \frac{\exp (\bar{\varepsilon})-1}{\exp (\bar{\varepsilon})+\frac{\underline{\underline{M}}_{i}}{\bar{M}_{i}}} \rho_{i}(t) \leq e_{i}(t) \leq \bar{M}_{i} \frac{\exp (\bar{\varepsilon})-1}{\exp (\bar{\varepsilon})+\frac{\bar{M}_{i}}{\underline{M}_{i}}} \rho_{i}(t)<\bar{M}_{i} \rho_{i}(t)$

for $i \in\left\{f, y, z, o_{1}, o_{2}, o_{3}\right\}$ and for all $t \geq 0$, which completes the proof.
315 Remark 1. From the aforementioned proof, it is worth noticing that the proposed control scheme does not incorporate any information regarding the matrices $\boldsymbol{M}, \boldsymbol{C}, \boldsymbol{D}, \mathrm{g}$, the deformation model $\Phi(\chi)$ or the external disturbances $\delta(t)$, which all affect only the size of $\bar{\varepsilon}$ and $\bar{\varepsilon}_{\zeta}$ but leave unaltered the achieved convergence properties as (39) dictates. In fact, the actual transient and steady state performance is determined by the selection of the performance functions $\rho_{i}(t)$ and performance parameters $M_{i}, \bar{M}_{i}, i \in\left\{f, y, z, o_{1}, o_{2}, o_{3}\right\}$. More specifically, for any initial force/position/orientation tracking error, the performance functions $\rho_{i}(t)$ and parameters $\underline{M}_{i}, \bar{M}_{i}, i \in$ $\left\{f, y, z, o_{1}, o_{2}, o_{3}\right\}$ are selected such that: $\left.i\right)-\underline{M}_{i}<e_{i}(0)<\bar{M}_{i}$ and $i$ ) the desired transient and steady state performance specifications are incorporated.

Remark 2. It should be noted that the selection of the control gains affects both the quality of evolution of the errors $e_{i}, i \in\left\{f, y, z, o_{1}, o_{2}, o_{3}\right\}$ inside the corresponding performance envelopes as well as the control input characteristics (e.g., decreasing the gain values leads to increased oscillatory behavior within the prescribed performance envelope described by (39), is improved when adopting higher values, enlarging, however, the control effort both in magnitude and rate). Additional fine tuning might be needed in real-time scenarios to retain the required control input signals within the feasible range that can be implemented by the actuators. Similarly, the control input constraints impose an upper bound on the required speed of convergence of $\rho_{i}(t), i \in\left\{f, y, z, o_{1}, o_{2}, o_{3}\right\}$. Hence, the selection of the control gains $k_{i}, i \in\left\{f, y, z, o_{1}, o_{2}, o_{3}\right\}$ and $\boldsymbol{K}_{\zeta}$ can have positive influence on the overall closed loop system response. More specifically, notice that (33) and (36) provide bounds on $\varepsilon(t)$ and $\varepsilon_{\zeta}(t)$. Therefore, invoking (15) and (24) we can select the control gains $k_{i}, i \in\left\{f, y, z, o_{1}, o_{2}, o_{3}\right\}$ and $\boldsymbol{K}_{\zeta}$ such that $\zeta^{r}$ and $\tau$ are retained within certain bounds. Nevertheless, (33) and (36) involve via the terms $\boldsymbol{B}_{x}$ and $\boldsymbol{B}_{\zeta}$ the parameters of the model, the external disturbances and the desired performance specifications. Thus, an upper bound of the dynamic parameters of the system as well as of the exogenous disturbances should be given in order to extract any relationships between the achieved performance and the input constraints. Finally, in the same vein, the selection of the velocity performance functions $\rho_{\zeta_{i}}(t), i=1, \ldots, n$ affects similarly both the evolution of the force/position/orientation errors within the corresponding performance envelopes as well as the control input characteristics.

\section{Results}

In this section, the theoretical findings are verified via both simulation and experimental studies. The simulation results were conducted using a dynamic simulation environment built in MATLAB ${ }^{\circledR}$. The experimental results were conducted in a test tank employing a small UVMS.

\subsection{Simulation study}

The UVMS model considered in the simulation is an AUV equipped with a small 4 DoFs manipulator attached at the bow 
of the vehicle (see Fig 3). The design parameters of the AUV and the robotic manipulator are given in Tables 1, 3, The dynamic equations of UVMS were derived based on the NewtonEuler approach [29] and their simulation was carried out using a dynamic simulation environment built in MATLAB ${ }^{\circledR}$ with sampling time $0.1 \mathrm{sec}$, which is common in a real time operation with an underwater robotic system. We considered a scenario involving 3D motion, where the end-effector of the UVMS is in compliant contact with a planar surface with stiffness model $f=k \chi^{2}, k=300 \frac{\mathrm{N}}{\mathrm{m}^{2}}$ which is unknown to the controller. The initial configuration is depicted in Fig 3 . We as-

Table 1: Vehicle parameters

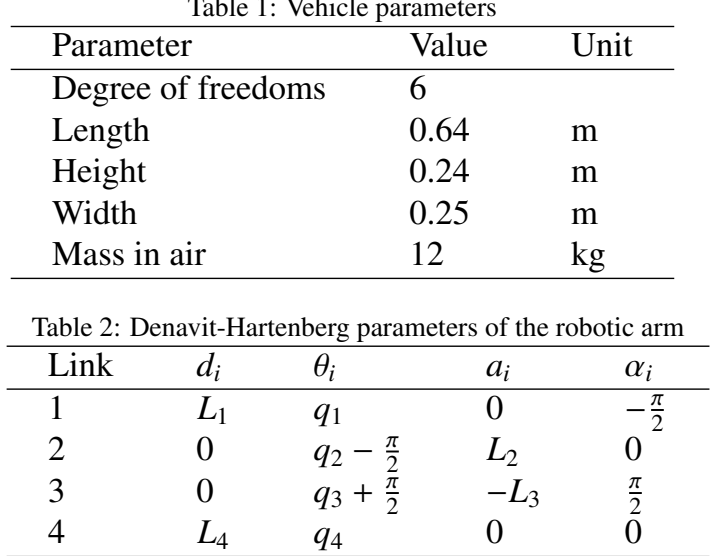

Table 3: Parameters of the robotic arm

\begin{tabular}{lll}
\hline Parameter & Value & Unit \\
\hline Link 1 Length $\left(L_{1}\right)$ & 0.077 & $\mathrm{~m}$ \\
Link 2 Length $\left(L_{2}\right)$ & 0.147 & $\mathrm{~m}$ \\
Link 3 Length $\left(L_{3}\right)$ & 0.028 & $\mathrm{~m}$ \\
Link 4 Length $\left(L_{4}\right)$ & 0.075 & $\mathrm{~m}$ \\
Link 1 Mass & 0.1 & $\mathrm{~kg}$ \\
Link 2 Mass & 0.2 & $\mathrm{~kg}$ \\
Link 3 Mass & 0.1 & $\mathrm{~kg}$ \\
Link 4 Mass & 0.12 & $\mathrm{~kg}$ \\
Link Diameter & 6 & $\mathrm{~cm}$
\end{tabular}

sumed that the UVMS is in contact with the compliant environment exerting a force normal to the surface $f(0)=0.5 \mathrm{~N}$. The desired force profile along the normal to the surface direction is set as $f^{d}(t)=4 N$. Moreover, the UVMS should track a desired position trajectory on the surface and attain a perpendicular orientation (i.e., $\boldsymbol{R}^{d}=I_{3 \times 3}$ ) with respect to the surface. The constants $\underline{f}^{*}$ and $\bar{f}^{*}$ are chosen as $\underline{f}^{*}=0.1 \mathrm{~N}$ and $\bar{f}^{*}=5 N$ in order to ensure a priori that the contact with ${ }_{415}$ the surface is never lost and that excessive interaction forces are avoided. In this spirit, as it was described earlier, we set $\underline{M}_{f}=3.9, \bar{M}_{f}=1$. All other performance parameters at the kinematic level are given in Table-4 Moreover, the control gains were selected as $k_{i}=0.2, j \in\left\{f, p_{1}, p_{2}, o_{1}, o_{2}, o_{3}\right\}$. The 420 design parameters regarding to the second level were chosen as: $\rho_{\zeta_{i}}^{0}=1.0, \boldsymbol{K}_{\zeta}=2 I_{n \times n}$. Notice that the dynamic parameters of the UVMS as well as the stiffness of the planar surface

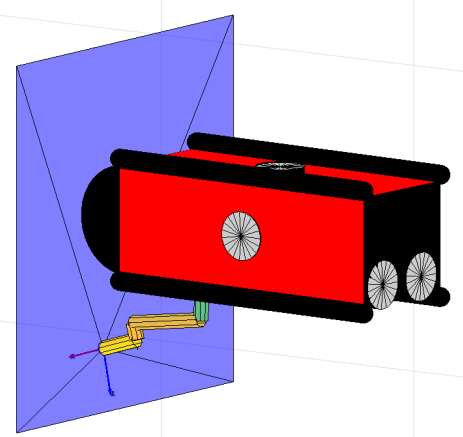

Figure 3: Workspace including the UVMS in compliant contact with a planar surface.

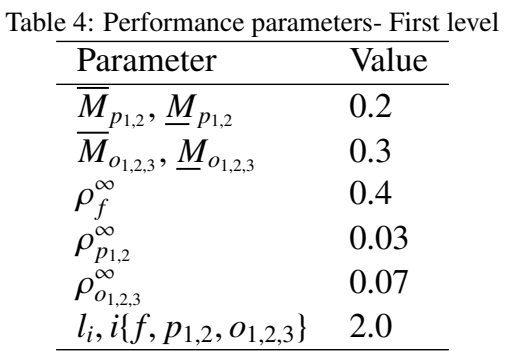

were considered unknown for the controller. Furthermore, the secondary task velocities (18) were designed appropriately to avoid the violation of the manipulator joint limits. However, more complicated secondary tasks (e.g., manipulability) could also be incorporated following the task priority based control techniques [48, 66]. In addition, in order to test the robustness of the proposed scheme, in the subsequent simulation study the dynamics of the UVMS were affected by external disturbances in the form of slowly time varying sea currents acting along $x, y$ and $z$ axes modeled by the corresponding velocities $v_{i}^{\{I\}}=0.1 \sin \left(\frac{\pi}{25} t\right) \frac{m}{s}, i \in\{x, y, z\}$. Finally, bounded measurement noise of normal distribution with $5 \%$ standard deviation was considered during the simulation study.

The results are depicted in Fig 4.Fig.7] The evolution of the position trajectory on the surface is presented in Fig 4 . It can be easily observed that the actual position of the end-effector (indicated by red color) converges to the desired one (indicated by green color) and follows the desired trajectory profile. Fig 5 presents the evolution of the actual force exerted by the UVMS with respect to the desired force profile. Moreover, it can be seen that the force exerted by the UVMS remained inside the desired region, securing that the contact is never lost and the exerted force never overshoots the predefined value. The evolution of the errors at the first and second level of the proposed controller are illustrated in Fig 6 and Fig 7 , respectively. It can be concluded that even with the influence of external disturbances as well as measurements noise, the errors in all directions converge close to zero and remain bounded below and above by the corresponding performance functions. 


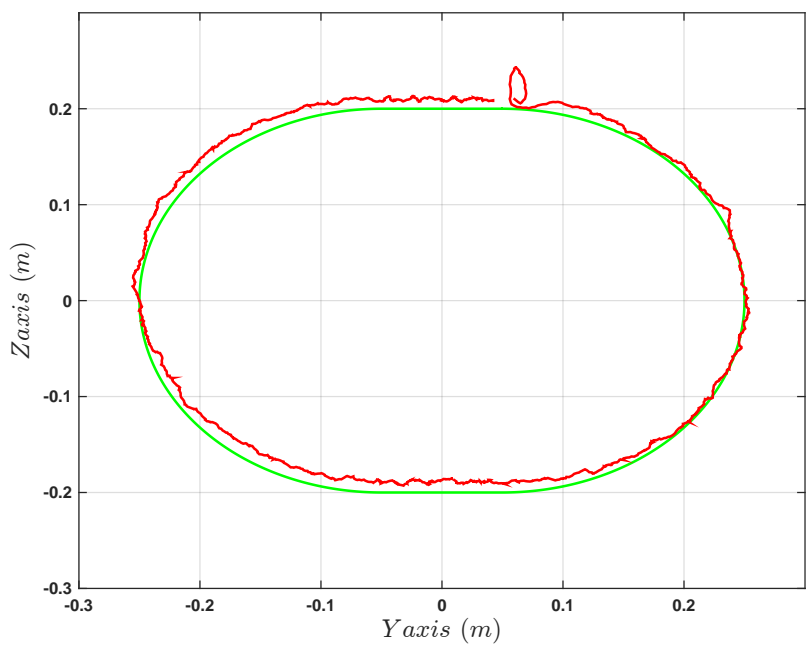

Figure 4: The evolution of the position trajectory on the surface. The desired trajectory and the actual position of the end-effector on the surface are indicated by green and red color respectively. The end-effector position converges and follows the desired trajectory profile.

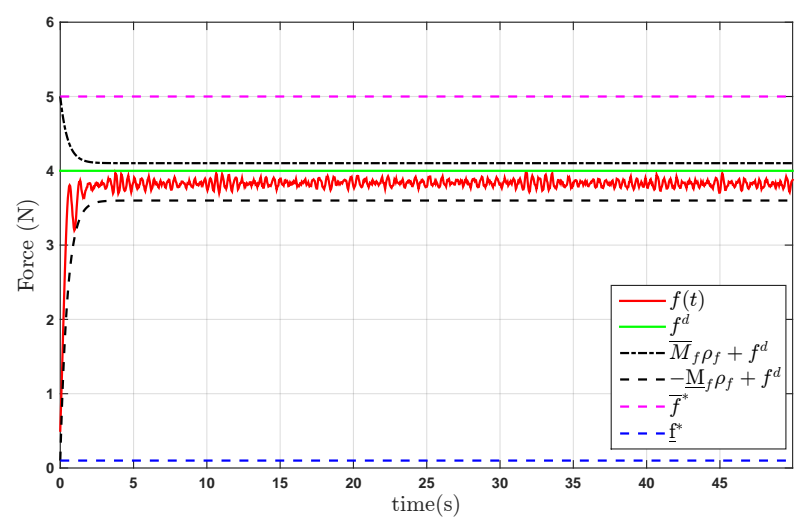

Figure 5: The evolution of the force trajectory. The desired constant force and the actual force exerted by the UVMS are indicated by green and red color respectively.

\subsection{Experimental Results}

This section demonstrates the efficacy of the proposed position/force control scheme via an experimental procedure with a small UVMS.

\subsubsection{Experimental Setup}

The experiments were carried out inside the NTUA, Control Systems Lab test tank, with dimensions $5 m \times 3 m \times 1.5 m$ (Fig. 8). In the bottom of the tank, sparse visual features have been added in order to improve optical-flow velocity estimations. The vehicle used in this work is a 4 DoFs Seabotix LBV [67], actuated in Surge, Sway, Heave and Yaw via a 4 thruster set configuration. The vehicle is equipped with a small custom-made 4 DoFs underwater manipulator, with revolute 445 joints and a compliant (spring-based) end-effector. The system is equipped with two cameras: i) a down-looking Sony PlayStation Eye camera, with $640 \times 480$ pixels at 30 frames per second (fps) enclosed in a waterproof housing and ii) a forward looking camera (Seabotix LBV default camera) with $640 \times 480$ pixels at 450 25 frames per second (fps). The vehicle is also equipped with an $S B G I G-500 A$ AHRS, delivering temperature-compensated
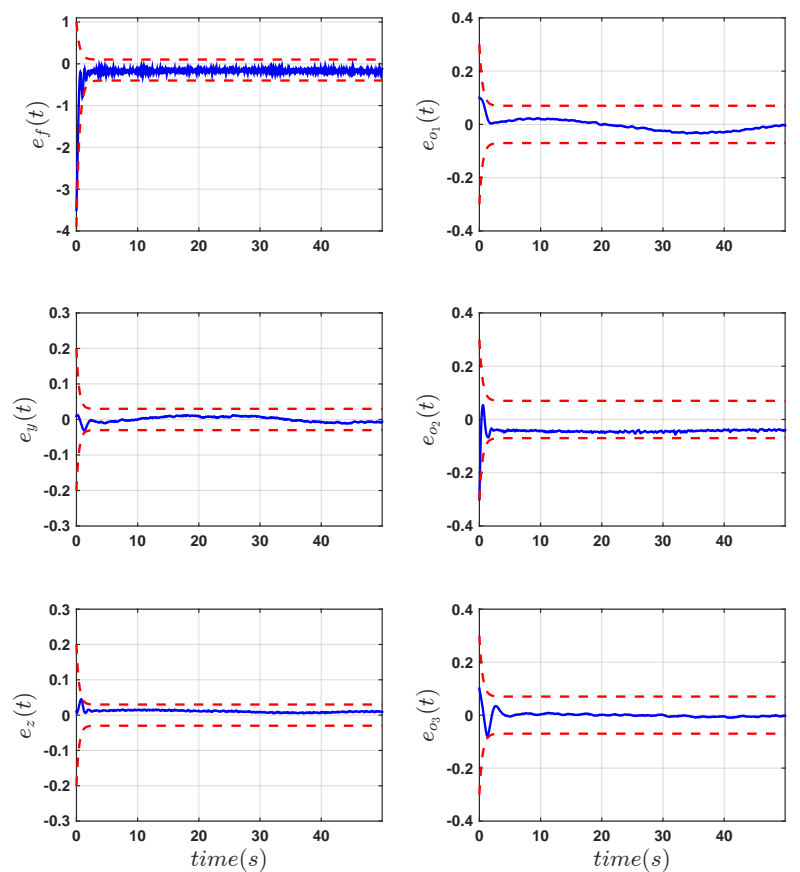

Figure 6: The evolution of the errors at the first level of the proposed control scheme. The errors and performance bounds are indicated by blue and red color respectively.
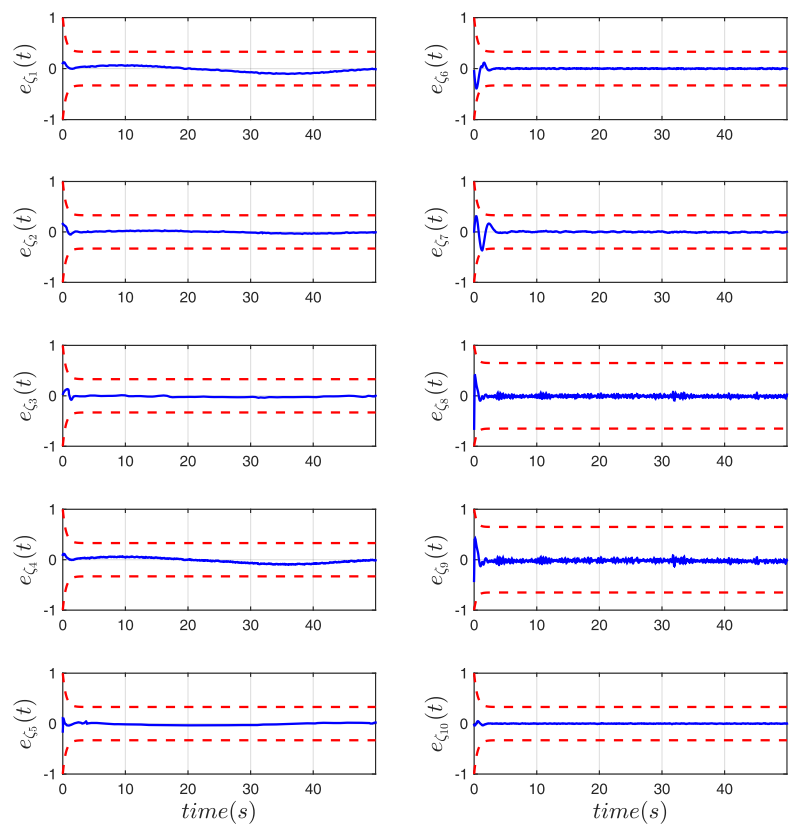

Figure 7: The evolution of the errors at the second level of the proposed control scheme. The errors and performance bounds are indicated by blue and red color respectively.

$3 \mathrm{D}$ acceleration, angular velocity and orientation measurements at $100 \mathrm{~Hz}$. Finally, a marker localization system based on the ArUco library [68] is employed in order to determine the pose of the vehicle with respect to the panel (see Fig. 9). A state estimation algorithm based on the Complementary Filters notion, as in [69], delivers the pose, velocity and acceleration estimates of the underwater vehicle by fusing data from the available onboard sensors while the position and velocity of the arm joints are available via the on-board encoders. The force measure- 


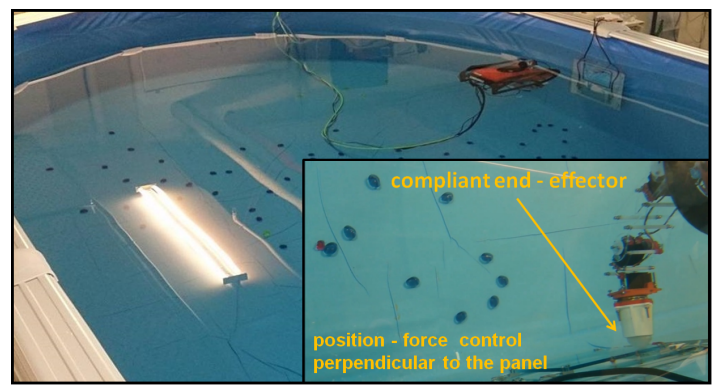

Figure 8: The NTUA, Control Systems Lab test tank and Position-Force Control Scenario

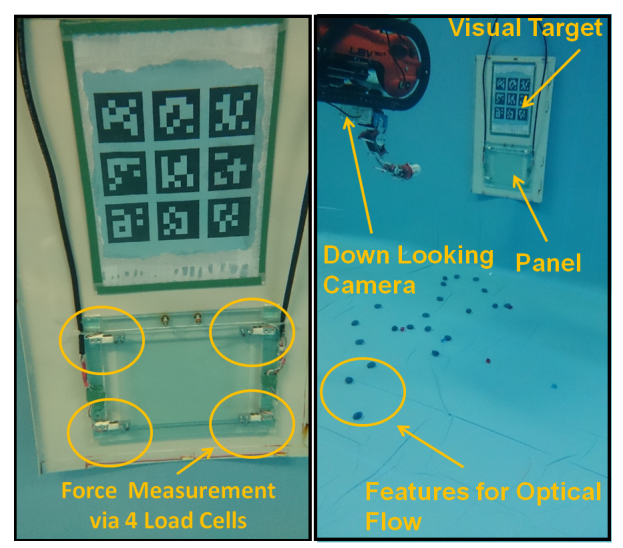

Figure 9: a) Load cells mounted on the panel, b)Visual features for state estimation

ments along the desired direction (perpendicular to the panel) are acquired by 4 load-cells, properly mounted on the base corners of the panel, as shown in Fig. 9. At each time instant, the sum of the 4 load-cells is incorporated as force feedback to the system. The load-cells are connected directly to the control PC, via a PhidgetBridge data I/O device. Finally, the software implementation of the state estimation algorithm as well as of the proposed position/force control scheme was conducted in $\mathrm{C}++$ and Python under the Robot Operating System (ROS) [70].

\subsubsection{Experimental results}

This subsection demonstrates experimental results obtained by the proposed position/force control scheme. In this scenario, the UVMS end-effector should follow a line trajectory along the panel, while maintaining a normal constant force to the panel. We consider that the end-effector has already achieved contact with the panel. The desired force is set to $f^{d}=5 \mathrm{~N}$, while the end-effector must perform a periodical motion between $-0.05 \mathrm{~m}$ and $0.05 \mathrm{~m}$ along the panel $y$ local axis, while the position along $z$ local axis should remain constant. The state responses are shown in Fig. 10, 12, while the trajectory of the end-effector on the plane is depicted in Fig. 13, As it can be easily observed, the proposed position/force control scheme fulfilled the interaction task successfully.

\section{Video}

A video demonstrating the aforementioned experimental result of the proposed methodology can be found in a HD video at the following url: https://youtu.be/PCHLsUb-vMO

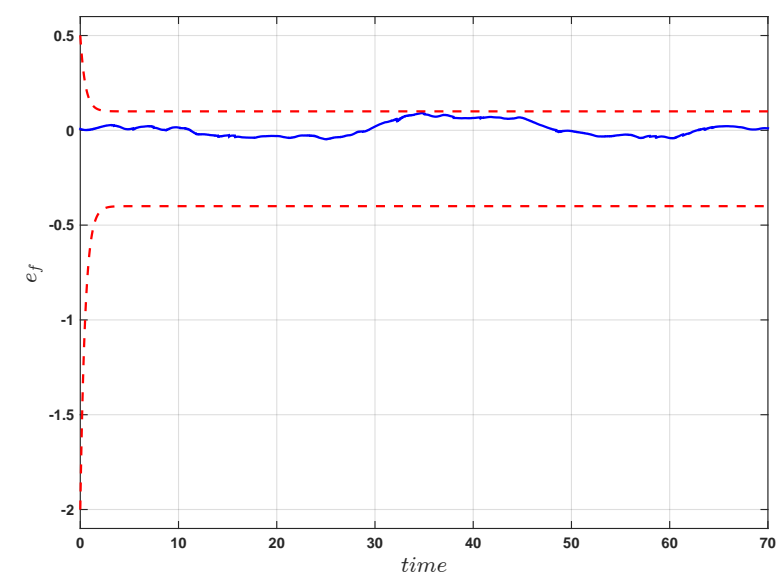

Figure 10: The evolution of the force error during the experiment. Perpendicular force $f$ along $x$ panel local axis.
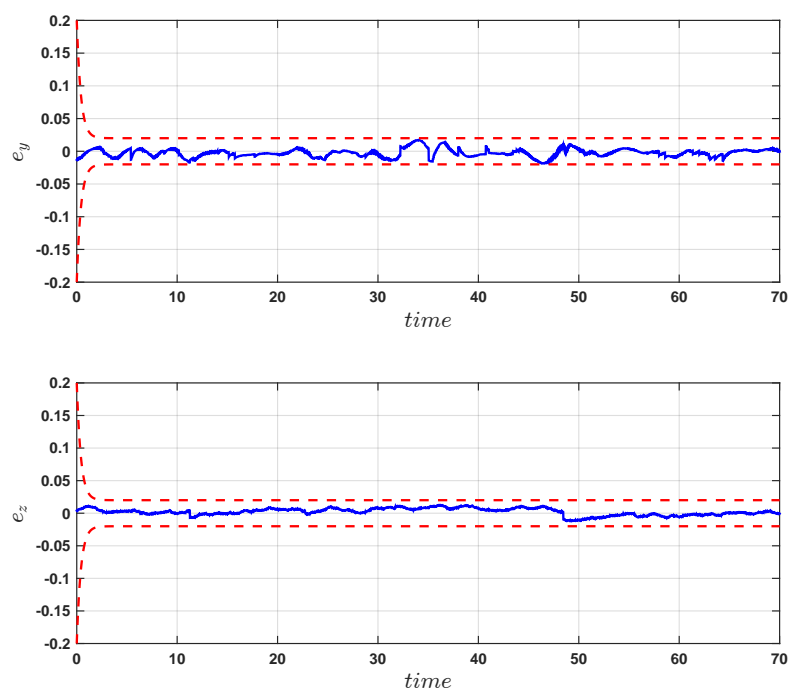

Figure 11: The evolution of the end-effector position errors along $y$ and $z$ direction during the experiment.
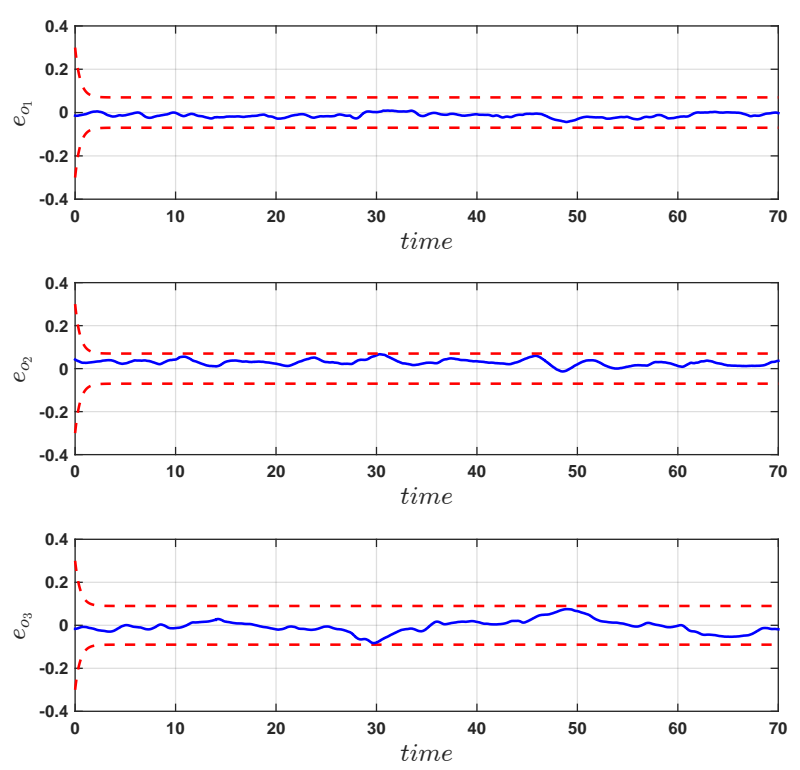

Figure 12: The evolution of the end-effector orientation errors direction during the experiment. 


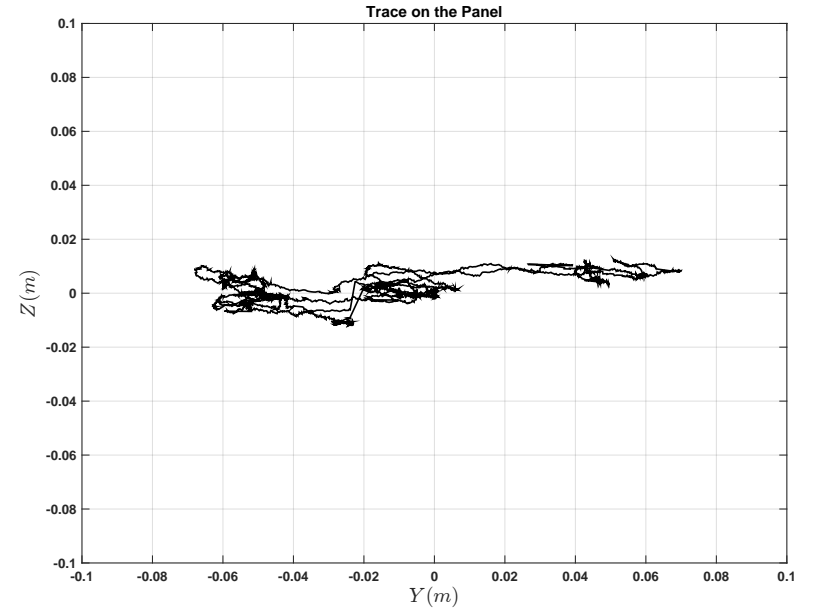

Figure 13: The trajectory of the end-effector w.r.t the horizontal plane. Perpendicular force is kept constant at all times.

\section{Conclusions}

This work presents a robust force/position control scheme for ${ }^{540}$ a UVMS in compliant contact with the environment, with great applications in underwater robotics (e.g. sampling of the sea organisms, underwater welding, object handling). The proposed control scheme does not required any a priori knowledge of the ${ }^{545}$ UVMS dynamical parameters or the stiffness model. It guarantees a predefined behavior in terms of desired overshoot, transient and steady state response and it is robust with respect to external disturbances and measurement noises. Moreover, the ${ }^{550}$ proposed controller exhibits the following important characteristics: i) it is of low complexity and thus can be easily used in most UVMSs ii) the performance of the proposed scheme (e.g., desired overshoot, steady state response) is a priori and explic- ${ }^{555}$ itly imposed by certain designer-specified performance functions, and is fully decoupled by the control gains selection, thus simplifying the control design.

\section{Acknowledgments}

This work was supported by the "ROBOCADEMY", Maries65 Curie ITN Grant Agreement no FP7-608096 funded by the EU action " $7^{\text {th }}$ Framework Programme - The 2013 People Work Programme - EC Call Identifier FP7-PEOPLE- 2013-ITN, Implementation Mode: Multi-ITN".

\section{References}

[1] T. Fossen, Guidance and control of ocean vehicles, Wiley, New York.

[2] P. Ridao, M. Carreras, D. Ribas, P. Sanz, G. Oliver, Intervention auvs: The next challenge, Annual Reviews in Control 40 (2015) 227-241.

[3] F. Bruno, M. Muzzupappa, L. Barbieri, A. Gallo, G. Ritacco, A. Lagudi, M. F. La Russa, S. A. Ruffolo, G. M. Crisci, M. Ricca, et al., The $\operatorname{comas}_{580}$ project: New materials and tools for improving the in situ documentation, restoration, and conservation of underwater archaeological remains, Marine Technology Society Journal 50 (4) (2016) 108-118.

[4] G. Antonelli, "Underwater Robots", Springer Tracts in Advanced Robotics, Springer International Publishing, 2013.
[5] H. Farivarnejad, S. Moosavian, Multiple impedance control for object manipulation by a dual arm underwater vehicle-manipulator system, Ocean Engineering 89 (2014) 82-98.

[6] H. Shim, B.-H. Jun, P.-M. Lee, H. Baek, J. Lee, Workspace control system of underwater tele-operated manipulators on an rov, Ocean Engineering 37 (11-12) (2010) 1036-1047.

[7] P. Londhe, S. Mohan, B. Patre, L. Waghmare, Robust task-space control of an autonomous underwater vehicle-manipulator system by pid-like fuzzy control scheme with disturbance estimator, Ocean Engineering 139 (2017) 1-13.

[8] S. Mohan, J. Kim, Coordinated motion control in task space of an autonomous underwater vehicle-manipulator system, Ocean Engineering 104 (2015) 155-167.

[9] G. Marani, S. Choi, J. Yuh, Underwater autonomous manipulation for intervention missions auvs, Ocean Engineering 36 (1) (2009) 15-23.

[10] E. Simetti, G. Casalino, Whole body control of a dual arm underwater vehicle manipulator system, Annual Reviews in Control 40 (2015) 191200.

[11] D. Lane, D. O’Brien, M. Pickett, J. Davies, G. Robinson, D. Jones, E. Scott, G. Casalino, G. Bartolini, G. Cannata, A. Ferrara, D. Angelleti, M. Coccoli, G. Veruggio, R. Bono, P. Virgili, M. Canals, R. Pallas, E. Gracia, C. Smith, Amadeus: Advanced manipulation for deep underwater sampling, IEEE Robotics and Automation Magazine 4 (4) (1997) 34-45.

[12] V. Rigaud, . Coste-Manière, M. Aldon, P. Probert, M. Perrier, P. Rives, D. Simon, D. Lane, J. Kiener, A. Casals, J. Amat, P. Dauchez, M. Chantler, Union: Underwater intelligent operation and navigation, IEEE Robotics and Automation Magazine 5 (1) (1998) 25-34.

[13] P. Sanz, P. Ridao, G. Oliver, G. Casalino, C. Insaurralde, C. Silvestre, C. Melchiorri, A. Turetta, Trident: Recent improvements about autonomous underwater intervention missions, IFAC Proceedings Volumes (IFAC-PapersOnline) 3 (PART 1) (2012) 355-360.

[14] M. Prats, J. Garcia, S. Wirth, D. Ribas, P. Sanz, P. Ridao, N. Gracias, G. Oliver, Multipurpose autonomous underwater intervention: A systems integration perspective, 2012 20th Mediterranean Conference on Control and Automation, MED 2012 - Conference Proceedings (2012) 1379_ 1384.

[15] J. Fernández, M. Prats, P. Sanz, J. García, R. Marín, M. Robinson, D. Ribas, P. Ridao, Grasping for the seabed: Developing a new underwater robot arm for shallow-water intervention, IEEE Robotics and Automation Magazine 20 (4) (2013) 121-130.

[16] E. Simetti, G. Casalino, S. Torelli, A. Sperindé, A. Turetta, Floating underwater manipulation: Developed control methodology and experimental validation within the trident project, Journal of Field Robotics 31 (3) (2014) 364-385.

[17] M. Prats, D. Ribas, N. Palomeras, J. García, V. Nannen, S. Wirth, J. Fernández, J. Beltrán, R. Campos, P. Ridao, P. Sanz, G. Oliver, M. Carreras, N. Gracias, R. Marín, A. Ortiz, Reconfigurable auv for intervention missions: A case study on underwater object recovery, Intelligent Service Robotics 5 (2012) 19-31.

[18] D. Ribas, P. Ridao, A. Turetta, C. Melchiorri, G. Palli, J. Fernandez, P. Sanz, I-auv mechatronics integration for the trident $\mathrm{fp} 7$ project, IEEE/ASME Transactions on Mechatronics 20 (5) (2015) 2583-2592.

[19] D. Lane, F. Maurelli, P. Kormushev, M. Carreras, M. Fox, K. Kyriakopoulos, Persistent autonomy: The challenges of the pandora project, IFAC Proceedings Volumes (IFAC-PapersOnline) 9 (PART 1) (2012) 268-273.

[20] A. Carrera, N. Palomeras, D. Ribas, P. Kormushev, M. Carreras, An intervention-auv learns how to perform an underwater valve turning, OCEANS 2014.

[21] A. Carrera, N. Palomeras, N. Hurtos, P. Kormushev, M. Carreras, Learning multiple strategies to perform a valve turning with underwater currents using an i-auv, MTS/IEEE OCEANS 2015 - Genova: Discovering Sustainable Ocean Energy for a New World.

[22] J. Gancet, D. Urbina, P. Letier, M. Ilzokvitz, P. Weiss, F. Gauch, G. Antonelli, G. Indiveri, G. Casalino, A. Birk, M. Pfingsthorn, S. Calinon, A. Tanwani, A. Turetta, C. Walen, L. Guilpain, Dexrov: Dexterous undersea inspection and maintenance in presence of communication latencies, IFAC-PapersOnLine 28 (2) (2015) 218-223.

[23] D. Rizzo, F. Bruno, L. Barbieri, M. Muzzupappa, Kinematic performances evaluation of a hydraulic underwater manipulator, in: OCEANS 2017-Aberdeen, IEEE, 2017, pp. 1-6. 
24] F. Zhang, G. Marani, R. Smith, H. Choi, Future trends in marine robotics, IEEE Robotics and Automation Magazine 22 (1) (2015) 14-21 and 122.

[25] G. Marani, S. Choi, J. Yuh, Real-time center of buoyancy identification for optimal hovering in autonomous underwater intervention, Intelligent Service Robotics 3 (3) (2010) 175-182.

[26] M. Dunnigan, G. Russell, Evaluation and reduction of the dynamic coupling between a manipulator and an underwater vehicle, IEEE Journal of Oceanic Engineering 23 (3) (1998) 260-273.

[27] B. Siciliano, L. Sciavicco, L. Villani, Robotics: modelling, planning and control., Springer Verlag.

[28] J. Yuh, Design and control of autonomous underwater robots: A survey, Autonomous Robots 8 (1) (2000) 7-24.

[29] I. Schjølberg, T. I. Fossen, Modelling and control of underwater vehiclemanipulator systems, in: in Proc. rd Conf. on Marine Craft maneuvering and control, Citeseer, 1994.

[30] T. J. Tarn, G. Shoults, S. Yang, A dynamic model of an underwater vehicle with a robotic manipulator using kane's method, Autonomous Robots 3 (2) (1996) 269-283.

[31] S. McMillan, D. E. Orin, R. B. McGhee, Efficient dynamic simulation of an underwater vehicle with a robotic manipulator, IEEE Transactions on 675 Systems, Man, and Cybernetics 25 (8) (1995) 1194-1206.

[32] M. Dunnigan, D. Lane, A. Clegg, I. Edwards, Hybrid position/force control of a hydraulic underwater manipulator, Control Theory and Applications, IEE Proceedings - 143 (2) (1996) 145-151.

[33] A. Clegg, M. Dunnigan, D. Lane, Self-tuning position and force control 680 of an underwater hydraulic manipulator, Robotics and Automation, 2001. Proceedings 2001 ICRA. IEEE International Conference on 4 (2001) 3226-3231 vol.4

[34] J.-H. Ryu, D.-S. Kwon, P.-M. Lee, Control of underwater manipulators mounted on an rov using base force information, Proceedings - IEEE In-685 ternational Conference on Robotics and Automation 4 (2001) 3238-3243.

[35] H. Kajita, K. Kosuge, Force control of robot floating on the water utilizing vehicle restoring force, Intelligent Robots and Systems, 1997. IROS '97., Proceedings of the 1997 IEEE/RSJ International Conference on 1 (1997) $162-167$ vol.1.

36] L. Lapierre, P. Fraisse, P. Dauchez, Position/force control of an underwater mobile manipulator, Journal of Robotic Systems 20 (12) (2003) 707-722.

[37] Y. Cui, T. Podder, N. Sarkar, Impedance Control of Underwater VehicleManipulator Systems (uvms), IEEE/RSJ International Conference on In-695 telligent Robots and Systems (1999) 148-153 vol.1.

[38] Y. Cui, N. Sarkar, A unified force control approach to autonomous underwater manipulation, Robotics and Automation, 2000. Proceedings. ICRA '00. IEEE International Conference on 2 (2000) 1263-1268 vol.2.

[39] G. Antonelli, S. Chiaverini, Task-priority redundancy resolution for un-700 derwater vehicle-manipulator systems, Proceedings - IEEE International Conference on Robotics and Automation 1 (1998) 768-773.

[40] D. R. Yoerger, J. G. Cooke, J.-J. E. Slotine, Influence of thruster dynamics on underwater vehicle behavior and their incorporation into control system design, IEEE Journal of Oceanic Engineering 15 (3) (1990) 167-178.705

[41] L. L. Whitcomb, D. R. Yoerger, Preliminary experiments in modelbased thruster control for underwater vehicle positioning, IEEE Journal of Oceanic Engineering 24 (4) (1999) 495-506.

[42] R. Bachmayer, L. L. Whitcomb, M. A. Grosenbaugh, An accurate fourquadrant nonlinear dynamical model for marine thrusters: Theory and ex-710 perimental validation, IEEE Journal of Oceanic Engineering 25 (1) (2000) $146-159$.

[43] A. Liegeois, Automatic supervisory control of the configuration and behavior of multibody mechanisms, IEEE transactions on systems, man, and cybernetics 7 (12) (1977) 868-871.

[44] O. Khatib, A unified approach for motion and force control of robot manipulators: The operational space formulation, IEEE Journal on Robotics and Automation 3 (1) (1987) 43-53.

[45] P. Baerlocher, R. Boulic, An inverse kinematics architecture enforcing an arbitrary number of strict priority levels, The visual computer 20 (6)720 (2004) 402-417.

[46] E. Olguin-Diaz, G. Arechavaleta, G. Jarquin, V. Parra-Vega, A passivity-based model-free force-motion control of underwater vehiclemanipulator systems, IEEE Transactions on Robotics 29 (6) (2013) 14691484.

[47] O. Kanoun, F. Lamiraux, P.-B. Wieber, Kinematic control of redun- dant manipulators: Generalizing the task-priority framework to inequality task, IEEE Transactions on Robotics 27 (4) (2011) 785-792.

[48] E. Simetti, G. Casalino, A novel practical technique to integrate inequality control objectives and task transitions in priority based control, Journal of Intelligent and Robotic Systems: Theory and Applications 84 (1-4) (2016) 877-902

[49] A. Escande, N. Mansard, P.-B. Wieber, Hierarchical quadratic programming: Fast online humanoid-robot motion generation, The International Journal of Robotics Research 33 (7) (2014) 1006-1028.

[50] S. Chiaverini, Singularity-robust task-priority redundancy resolution for real-time kinematic control of robot manipulators, IEEE Transactions on Robotics and Automation 13 (3) (1997) 398-410.

[51] N. Sarkar, T. Podder, Coordinated motion planning and control of autonomous underwater vehicle-manipulator systems subject to drag optimization, IEEE Journal of Oceanic Engineering 26 (2) (2001) 228-239.

[52] Z. Ismail, M. Dunnigan, Tracking control scheme for an underwater vehicle-manipulator system with single and multiple sub-regions and subtask objectives, IET Control Theory and Applications 5 (5) (2011) 721735.

[53] J. Han, J. Park, W. Chung, Robust coordinated motion control of an underwater vehicle-manipulator system with minimizing restoring moments, Ocean Engineering 38 (10) (2011) 1197-1206.

[54] S. Soylu, B. Buckham, R. Podhorodeski, Redundancy resolution for underwater mobile manipulators, Ocean Engineering 37 (2-3) (2010) 325343 .

[55] G. Antonelli, S. Chiaverini, N. Sarkar, External force control for underwater vehicle-manipulator systems, Robotics and Automation, IEEE Transactions on 17 (6) (2001) 931-938.

[56] S. Heshmati-alamdari, A. Nikou, K. Kyriakopoulos, D. Dimarogonas, A robust force control approach for underwater vehicle manipulator systems, IFAC-PapersOnLine 50 (1) (2017) 11197-11202

[57] K. L. Johnson, K. L. Johnson, Contact mechanics, Cambridge university press, 1987.

[58] S. Arimoto, P. T. A. Nguyen, H.-Y. Han, Z. Doulgeri, Dynamics and control of a set of dual fingers with soft tips, Robotica 18 (1) (2000) 71-80.

[59] F. Caccavale, C. Natale, B. Siciliano, L. Villani, Resolved-acceleration control of robot manipulators: A critical review with experiments, Robotica 16 (5) (1998) 565-573.

[60] L. Sciavicco, B. Siciliano, Modelling and control of robot manipulators, Springer Science \& Business Media, 2012

[61] C. Bechlioulis, G. Rovithakis, Prescribed performance adaptive control for multi-input multi-output affine in the control nonlinear systems, IEEE Transactions on Automatic Control 55 (5) (2010) 1220-1226.

[62] C. P. Bechlioulis, G. A. Rovithakis, Robust partial-state feedback prescribed performance control of cascade systems with unknown nonlinearities, IEEE Transactions on Automatic Control 56.

[63] C. Bechlioulis, G. Rovithakis, A low-complexity global approximationfree control scheme with prescribed performance for unknown pure feedback systems, Automatica 50 (4) (2014) 1217-1226.

[64] B. Siciliano, J. J. E. Slotine, A general framework for managing multiple tasks in highly redundant robotic systems, Advanced Robotics, 1991. 'Robots in Unstructured Environments', 91 ICAR., Fifth International Conference on (1991) 1211-1216 vol.2.

[65] E. D. Sontag, Mathematical Control Theory, Springer, London, U.K., 1998.

[66] E. Simetti, G. Casalino, Manipulation and transportation with cooperative underwater vehicle manipulator systems, IEEE Journal of Oceanic Engineering.

[67] Seabotix lbv150 URL http: //www . teledynemarine. com/lbv150-4/?BrandID=19

[68] S. Garrido-Jurado, R. M. noz Salinas, F. Madrid-Cuevas, M. MarínJiménez, Automatic generation and detection of highly reliable fiducial markers under occlusion, Pattern Recognition 47 (6) (2014) 2280 - 2292.

[69] P. Marantos, Y. Koveos, K. Kyriakopoulos, Uav state estimation using adaptive complementary filters, IEEE Transactions on Control Systems Technology 24 (4) (2016) 1214-1226.

[70] M. Quigley, B. Gerkey, K. Conley, J. Faust, T. Foote, J. Leibs, E. Berger, R. Wheeler, A. Ng, Ros: an open-source robot operating system, in: Proc. of the IEEE Intl. Conf. on Robotics and Automation (ICRA) Workshop on Open Source Robotics, Kobe, Japan, 2009. 